\title{
The Schwarzian equation for completely replicable functions
}

\author{
Abdelkrim El Basraoui and John McKay
}

\section{Abstract}

We describe the Schwarzian equations for the 328 completely replicable functions with integral $q$-coefficients [Ford et al., 'More on replicable functions', Comm. Algebra 22 (1994) no. 13, $5175-5193]$

\section{Introduction}

We assume familiarity with $[\mathbf{1}, \mathbf{3}, \mathbf{7}, \mathbf{1 3}, \mathbf{1 4}]$.

In 1877, Dedekind [4] gave the Schwarzian equation

$$
\{\lambda, z\}+\frac{\lambda^{2}-\lambda+1}{\lambda^{2}(1-\lambda)^{2}} \lambda^{\prime 2}=0,
$$

for the principal modulus, $\lambda$, on $\Gamma(2)$. He even defined the $j$-function by its Schwarzian equation

$$
\{j, z\}+\frac{36 j^{2}-41 j+32}{72 j^{2}(1-j)^{2}} j^{\prime 2}=0,
$$

which loses its mystery when expanded as a partial fraction that describes its fundamental domain (see (6)).

It is useful to define:

- the arithmetic $j$-function

$$
j_{\text {arith }}(z)=\frac{1}{q}+744+196884 q+\ldots \quad \text { for } q=e^{2 \pi i z}, z \in \mathfrak{H}^{+}
$$

- the monster $j$-function

$$
j_{\text {monst }}(z)=j_{\operatorname{arith}}(z)-744
$$

and

- the analytic $j$-function

From the Schwarzian equation

$$
j_{\text {anal }}(z)=\frac{1}{1728} j_{\text {arith }}(z)
$$

$$
\{f, z\}+R(f) f^{\prime 2}=0,
$$

we list $R(f)$ for all completely replicable $f$ with $q$-coefficients in $\mathbb{Z}$.

\section{Preliminaries: the Schwarz derivative}

\subsection{Definition and properties}

The Schwarz derivative is a differential operator defined for a meromorphic function $f$ on a domain $D$ of $\mathbb{C}$ by

$$
\{f, z\}=2\left(\frac{f^{\prime \prime}}{f^{\prime}}\right)^{\prime}-\left(\frac{f^{\prime \prime}}{f^{\prime}}\right)^{2}=2 \frac{f^{\prime \prime \prime}}{f^{\prime}}-3 \frac{f^{\prime \prime 2}}{f^{\prime 2}}
$$

Received 22 April 2014; revised 9 May 2017.

2010 Mathematics Subject Classification 11F03, 11F22 (primary), $20 \mathrm{D} 08$ (secondary). 
where $f^{\prime}=(1 / 2 \pi i)(d f / d z)$. This was used by Poincaré in his 1882 paper Mémoire sur les fonctions fuchsiennes [12]. It also appears in $[\mathbf{5}, \mathbf{8}, \mathbf{1 0}, \mathbf{1 1}]$.

We give some properties of the Schwarz derivative (see [9]).

- For all $\left(\begin{array}{ll}a & b \\ c & d\end{array}\right) \in \mathrm{GL}_{2}^{+}(\mathbb{C}),\{(a f+b) /(c f+d), z\}=\{f, z\}$.

- $\{f, z\}=\{g, z\}$ if and only if each is a linear fraction of the other.

- Consequently, $\{f, z\}=0$ if and only if $f=(a z+b) /(c z+d)$ for some $\left(\begin{array}{ll}a & b \\ c & d\end{array}\right) \in \mathrm{GL}_{2}^{+}(\mathbb{C})$.

- It is a quadratic differential. If $u$ is a function of $z$, then

$$
\{f, z\} d z^{2}=\{f, u\} d u^{2}+\{u, z\} d z^{2} .
$$

- In particular, for $a, b, c, d$, as above, and $u=(a z+b) /(c z+d)$,

$$
\{f, u\}=\frac{(c z+d)^{4}}{a d-b c}\{f, z\} .
$$

- Inversion formula. If $f^{\prime}\left(z_{0}\right) \neq 0$ for some point $z_{0}$, then, in a neighborhood of $z_{0}$,

$$
\{z, f\}=-(d z / d f)^{2}\{f, z\} .
$$

\subsection{Link with differential equations}

One of our motivations for this paper is the role played by the Schwarz derivative in differential equations. In fact, there is a close relationship between the Schwarz derivative and second-order linear differential equations that can be illustrated as follows. Let $R(z)$ be a meromorphic function on a domain $D$ of $\mathbb{C}$. Then the (local) solutions of the second-order linear differential equation

$$
y^{\prime \prime}(z)+R(z) y(z)=0
$$

are two-dimensional vector spaces, and if $y_{1}$ and $y_{2}$ are two linearly independent solutions, then the quotient $f=y_{1} / y_{2}$ is a solution of the Schwarzian equation

$$
\{z, f\}=-R(z)
$$

Conversely, if $f$ is a solution to (2), then $y_{1}=f / \sqrt{f^{\prime}}, y_{2}=1 / \sqrt{f^{\prime}}$ are two linearly independent solutions to (1).

Furthermore, if we set $u=f^{\prime \prime} / f^{\prime}$, then from (1) we obtain the Riccati differential equation

$$
2 u^{\prime}-u^{2}=R(z)
$$

\section{Modular Schwarzian equations}

In this section, we establish the Schwarzian equation that we used to compute $R(f)$.

\subsection{Schwarz derivative of modular functions}

In [10], it was shown that the Schwarz derivative can have an important role in the theory of modular forms as well. The following proposition is an example.

Proposition 3.1 [10]. (i) If $f$ is a modular function for a discrete subgroup $G$ (of the first kind) of $\mathrm{PSL}_{2}(\mathbb{R})$, then $\{f, z\}$ is a weight four modular form for $G$.

(ii) If $\Gamma$ is of genus zero and $f_{\Gamma}$ is a principal modulus for $\Gamma$, then $\left\{f_{\Gamma}, z\right\}$ is a weight four modular form for the normalizer of $\Gamma$ inside $\mathrm{PSL}_{2}(\mathbb{R})$. 
The first statement is an immediate consequence of the chain rule, while the second statement is, however, less trivial.

One result that is of importance to us is the following proposition from [10].

Proposition 3.2. Let $\Gamma$ and $f_{\Gamma}$ be as in Proposition 3.1. Then $\{f, z\}$ has a pole of order two at elliptic points of $\Gamma$ and is holomorphic everywhere including at cusps.

The following is a corollary of this.

COROLlary 3.3. If $\Gamma$ has no elliptic points, then $\{f, z\}$ is a holomorphic weight four modular form for $\Gamma$.

REMARKS 3.1.

- Note that $\{z, f\}$ is a modular function. However, its invariance group is, in general, not larger than $\Gamma$.

- The maximum number of elliptic points for moonshine groups is at most 26 (including $\infty)$

\subsection{Schwarzian equations}

Let $f$ be a modular function for a Fuchsian group $\Gamma$ of first kind.

THEOREM 3.4 [6]. If $f$ is non-constant, then the (multi-valued) inverse function $z=z(f)$ can be expressed as the quotient of two (linearly independent) solutions of the differential equation

$$
\frac{d^{2} y}{d f^{2}}+R(f) y=0
$$

where $R$ is an algebraic function of $f$. Moreover, if $f$ has a single pole in the fundamental domain of $\Gamma$, then $R$ is a rational function of $f$.

Now, from the results of $\S 2.2$, it follows that

$$
\{z, f\}=R(f)
$$

and using the inversion formula,

$$
\{f, z\}=-R(f) f^{\prime 2} .
$$

For $\Gamma$ of genus zero, we deduce a similar type of equation involving the principal modulus for $\Gamma$. Indeed, let $f_{\Gamma}$ denote the principal modulus for $\Gamma$. Then, on the one hand, using the chain rule property for the Schwarz derivative, we see that $\{f, z\}$ is a weight four modular form for $\Gamma$. On the other hand, an easy computation shows that $f^{\prime}$ is a weight two modular form for $\Gamma$, so that $f^{\prime 2}$ is of weight two. Hence the quotient $\{f, z\} / f^{\prime 2}$ is a modular function for $\Gamma$ which is of genus zero, and therefore it is a rational function in $f_{\Gamma}$.

In other words, $f$ satisfies the differential equation

$$
\{f, z\}+R\left(f_{\Gamma}\right) f^{\prime 2}=0,
$$

where $R\left(f_{\Gamma}\right)$ is a rational function in $f_{\Gamma}$. In particular, for $f=f_{\Gamma}$, every principal modulus satisfies the Schwarzian equation

$$
\left\{f_{\Gamma}, z\right\}+R\left(f_{\Gamma}\right) f_{\Gamma}^{\prime 2}=0
$$

for some rational function $R$ in $f_{\Gamma}$. The inversion formula for the Schwarz derivative gives (3). 


\section{REMARK 3.1.}

- The above reasoning provides a direct proof in the case of genus zero groups only. In positive genus, this argument fails, simply because the field of modular functions is no longer generated by a single function.

- We conclude this subsection by noticing that the degree of $R\left(f_{\Gamma}\right)$, that is, the maximum degree of the numerator and denominator, is at most 52 by Proposition 3.1 as we are counting both elliptic points and $\infty$ as poles. This bound also holds for all completely replicable functions with integer coefficients, as our computation shows.

\subsection{A fundamental example}

In the introduction, we gave the example of the analytic $j$-function. We show the connection to Picard-Fuchs equations. We have seen that the $j$-function satisfies the Schwarzian equation

$$
\{f, z\}+\frac{36 j^{2}-41 j+32}{36 j^{2}(1-j)^{2}} f^{\prime 2}=0
$$

from which we deduce that

$$
R(j)=\frac{36 j^{2}-41 j+32}{36 j^{2}(1-j)^{2}}
$$

which has a partial fraction

$$
R(j)=\frac{1-\frac{1}{3^{2}}}{j^{2}}+\frac{1-\frac{1}{2^{2}}}{(1-j)^{2}}+\frac{1-\frac{1}{3^{2}}-\frac{1}{2^{2}}}{j(1-j)} .
$$

We see from this expression that the poles are located at the elliptic points $z=\rho$ and $z=i$.

Equation (5) has an equivalent formulation of hypergeometric type given by the PicardFuchs equation

$$
j(1-j) \frac{d^{2} y}{d j^{2}}+\left(\frac{2}{3}-\frac{7}{6} j\right) \frac{d y}{d j}-\frac{1}{144} j=0 .
$$

This example appears in [8], where Schwarz equations are used to find solutions of certain Halphen-type differential equations.

\section{Appendix}

In this appendix we list all rational modular functions for the class of completely replicable functions with integer coefficients as it appears in [7]. There are 328 such functions including the functions $1 / q+c q$ with $c \in\{0, \pm 1\}$.

For the three series of finite type $f(z)=1 / q+c q, c \in\{0, \pm 1\}$, the corresponding rational functions are

$$
R_{f}(Y)=Y^{2}-8 c .
$$

This can be established either by using our algorithm or simply by noticing that $f^{\prime}=f^{\prime \prime \prime}, f^{\prime \prime}=$ $f$ and $f^{\prime 2}=f^{2}-4 c$.

Our process for computing these rational functions relies on the Schwarz differential equation. For a completely replicable function $f$ given as a $q$-series with $R(f)=P(f) / Q(f)$ as its corresponding rational function, (4),

$$
\left(2 f^{\prime} f^{\prime \prime \prime}-3 f^{\prime \prime 2}\right) Q(f)+P(f) f^{\prime 4}=0 .
$$

We then use the $q$-expansion of $f$ to get a system of equations which we solve with respect to the coefficients of the polynomials $P$ and $Q$. For this purpose, one has to generate as many 
coefficients as needed and, for this reason, we use the Norton basis in [7]. One could also use the recurrence relations in $[\mathbf{1}]$. However, these relations involve the knowledge of the second replicate of each function. [N.B. $f=f^{(2)}$ for odd level function $f$.] This is to be found in [2] and in character tables computed in GAP.

To illustrate how the algorithm works, let us consider the following example of the monster $j$-function. The degree of $R(j)$, the rational function, is six because the modular group has two classes of elliptic points, namely, the classes of $z=i$ and $z=\rho$. Let $R(j)=P(j) / Q(j)$ be the corresponding rational function. Then

$$
\left(2 j^{\prime} j^{\prime \prime \prime}-3 j^{\prime \prime 2}\right) Q(j)+P(j) j^{\prime 4}=0 .
$$

If we write $P(Y)=\sum_{n=0}^{6} c_{n} Y^{n}, Q(Y)=\sum_{m=0}^{6} d_{m} Y^{m}$, using the $q$-expansion of the $j$-function we get a system of equations in the $c_{i}$ and $d_{l}$. The first 30 coefficients of the $j$-function are enough to solve for the corresponding rational function. We get infinitely many solutions $c_{0}=$ $1743552 c_{2}, c_{1}=-480 c_{2}, c_{2}=c_{2}, d_{0}=1071929106432 c_{2}, d_{1}=702812160 c_{2}, d_{2}=-2813184 c_{2}$, $d_{3}=-960 c_{2}, d_{4}=2 c_{2}, c_{3}=c_{4}=c_{5}=c_{6}=d_{5}=d_{6}=0$, and, after the substitution $Y=1 / Y$, we find $R(1 / j)$

$$
\frac{\left(1743552 Y^{2}-480 Y+1\right) Y^{2}}{(744 Y+1)^{2}(984 Y-1)^{2}}
$$

Table A.1 lists $R(f)$ for $f$ being a moonshine function while Table A.2 completes the list for the remaining completely replicable functions. We used the class names as in [7] to label each function. Also notice that, in Table A.1, following [3], we listed the McKay-Thompson series $25 \mathrm{z}, 49 \mathrm{z}$ and $50 \mathrm{z}$ even though these are not related to conjugacy classes of the Monster group.

TABLE A.1. $R(f)$ for 171 moonshine functions.

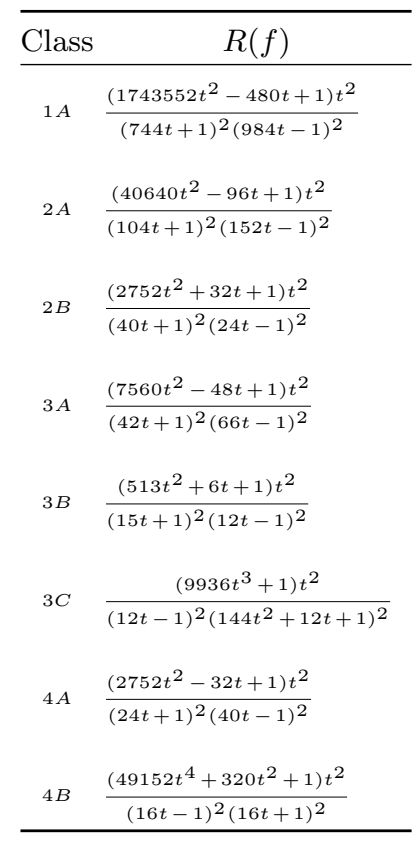


Table A.1. (Continued.)

\begin{tabular}{|c|c|}
\hline Class & $R(f)$ \\
\hline $4 C$ & $\frac{\left(1+192 t^{2}\right) t^{2}}{(8 t-1)^{2}(8 t+1)^{2}}$ \\
\hline $4 D$ & $\frac{\left(4096 t^{4}-64 t+1\right) t^{2}}{\left(64 t^{2}+1\right)^{2}}$ \\
\hline $5 A$ & $\frac{\left(359872 t^{4}+38096 t^{3}+848 t^{2}+8 t+1\right) t^{2}}{(16 t+1)^{2}\left(464 t^{2}+12 t-1\right)^{2}}$ \\
\hline $5 B$ & $\frac{\left(2497 t^{4}+1096 t^{3}+98 t^{2}+8 t+1\right) t^{2}}{(6 t-1)^{2}\left(29 t^{2}+10 t+1\right)^{2}}$ \\
\hline $6 A$ & $\frac{(120608 t+16352 t+492 t+4 t+1) t^{2}}{(10 t+1)^{2}(14 t+1)^{2}(22 t-1)^{2}}$ \\
\hline $6 B$ & $\frac{\left(117841 t^{4}+16804 t^{3}+486 t^{2}+4 t+1\right) t^{2}}{(12 t+1)^{2}\left(263 t^{2}+10 t-1\right)^{2}}$ \\
\hline $6 C$ & $\frac{\left(2848 t^{4}-800 t^{3}+108 t^{2}+4 t+1\right) t^{2}}{(10 t+1)^{2}(6 t-1)^{2}(2 t-1)^{2}}$ \\
\hline $6 D$ & $\frac{\left(1457 t^{4}+1124 t^{3}+6 t^{2}+4 t+1\right) t^{2}}{(4 t-1)^{2}\left(41 t^{2}+6 t+1\right)^{2}}$ \\
\hline $6 E$ & $\frac{\left(769 t^{4}+388 t^{3}+54 t^{2}+4 t+1\right) t^{2}}{(3 t+1)^{2}(4 t+1)^{2}(5 t-1)^{2}}$ \\
\hline $6 F$ & $\frac{\left(4096 t^{6}-304 t^{3}+1\right) t^{2}}{(4 t+1)^{2}\left(16 t^{2}-4 t+1\right)^{2}}$ \\
\hline $7 A$ & $\frac{\left(50328 t^{4}+8904 t^{3}+313 t^{2}+2 t+1\right) t^{2}}{(10 t+1)^{2}(9 t+1)^{2}(18 t-1)^{2}}$ \\
\hline $7 B$ & $\frac{\left(201 t^{4}+378 t^{3}+19 t^{2}+2 t+1\right) t^{2}}{(4 t-1)^{2}\left(13 t^{2}+5 t+1\right)^{2}}$ \\
\hline $8 A$ & $\frac{\left(22784 t^{4}+5120 t^{3}+224 t^{2}+1\right) t^{2}}{(8 t+1)^{2}\left(112 t^{2}+8 t-1\right)^{2}}$ \\
\hline $8 B$ & $\frac{\left(64 t^{2}-8 t+1\right)\left(64 t^{2}+8 t+1\right) t^{2}}{(8 t-1)^{2}(8 t+1)^{2}}$ \\
\hline $8 C$ & $\frac{\left(2816 t^{4}+1\right) t^{2}}{(4 t-1)^{2}(4 t+1)^{2}\left(16 t^{2}+1\right)^{2}}$ \\
\hline $8 D$ & $\frac{(4 t-1)^{2}(4 t+1)^{2} t^{2}}{\left(16 t^{2}+1\right)^{2}}$ \\
\hline $8 E$ & $\frac{\left(16 t^{2}+1\right)^{2} t^{2}}{(4 t-1)^{2}(4 t+1)^{2}}$ \\
\hline $8 F$ & $\frac{\left(4096 t^{8}-640 t^{4}+1\right) t^{2}}{\left(8 t^{2}-4 t+1\right)^{2}\left(8 t^{2}+4 t+1\right)^{2}}$ \\
\hline $9 A$ & $\frac{t^{2}\left(1+14337 t^{4}+3456 t^{3}+162 t^{2}\right)}{(6 t+1)^{2}\left(99 t^{2}+6 t-1\right)^{2}}$ \\
\hline $9 B$ & $\frac{(6 t+1)\left(36 t^{2}-6 t+1\right) t^{2}}{(3 t-1)^{2}\left(9 t^{2}+3 t+1\right)^{2}}$ \\
\hline
\end{tabular}


Table A.1. (Continued.)

\begin{tabular}{|c|c|}
\hline Class & $R(f)$ \\
\hline $10 \mathrm{~A}$ & $\frac{\left(10560 t^{4}+2256 t^{3}+128 t^{2}+1\right) t^{2}}{(12 t-1)^{2}(8 t+1)^{2}(4 t+1)^{2}}$ \\
\hline $10 B$ & $\frac{\left(3072 t^{6}-2304 t^{5}+1088 t^{4}-304 t^{3}+32 t^{2}+1\right) t^{2}}{(4 t-1)^{2}\left(16 t^{2}-4 t-1\right)^{2}}$ \\
\hline $10 \mathrm{C}$ & $\frac{\left(37 t^{2}-8 t+1\right)\left(5 t^{2}+8 t+1\right) t^{2}}{(2 t-1)^{2}\left(17 t^{2}+2 t+1\right)^{2}}$ \\
\hline $10 D$ & $\frac{\left(244897 t^{6}+159706 t^{5}+37683 t^{4}+3716 t^{3}+147 t^{2}+10 t+1\right) t^{2}}{(5 t+1)^{2}(6 t+1)^{2}\left(71 t^{2}+6 t-1\right)^{2}}$ \\
\hline $10 E$ & $\frac{\left(t^{2}+1\right)\left(13 t^{2}+6 t+1\right)\left(61 t^{4}+62 t^{3}+14 t^{2}-2 t+1\right) t^{2}}{(3 t-1)^{2}(2 t+1)^{2}(t+1)^{2}\left(5 t^{2}+2 t+1\right)^{2}}$ \\
\hline $11 \mathrm{~A}$ & $\frac{\left(115884 t^{6}+86896 t^{5}+23424 t^{4}+2620 t^{3}+112 t^{2}+8 t+1\right) t^{2}}{(6 t+1)^{2}\left(212 t^{3}+76 t^{2}+2 t-1\right)^{2}}$ \\
\hline $12 \mathrm{~A}$ & $\frac{\left(2848 t^{4}+800 t^{3}+108 t^{2}-4 t+1\right) t^{2}}{(2 t+1)^{2}(6 t+1)^{2}(10 t-1)^{2}}$ \\
\hline $12 B$ & $\frac{\left(769 t^{4}-388 t^{3}+54 t^{2}-4 t+1\right) t^{2}}{(5 t+1)^{2}(4 t-1)^{2}(3 t-1)^{2}}$ \\
\hline $12 \mathrm{C}$ & $\frac{\left(10368 t^{6}+336 t^{4}+32 t^{2}+1\right) t^{2}}{(6 t-1)^{2}(2 t+1)^{2}(2 t-1)^{2}(6 t+1)^{2}}$ \\
\hline $12 D$ & $\frac{\left(4096 t^{6}+304 t^{3}+1\right) t^{2}}{(4 t-1)^{2}\left(16 t^{2}+4 t+1\right)^{2}}$ \\
\hline $12 E$ & $\frac{\left(1152 t^{6}+720 t^{4}+1\right) t^{2}}{(2 t+1)^{2}(2 t-1)^{2}\left(12 t^{2}+1\right)^{2}}$ \\
\hline $12 F$ & $\frac{\left(t^{8}+28 t^{6}+1350 t^{4}+28 t^{2}+1\right) t^{2}}{\left(t^{2}+6 t+1\right)^{2}\left(t^{2}-6 t+1\right)^{2}}$ \\
\hline $12 G$ & $\frac{\left(6561 t^{8}-324 t^{6}-474 t^{4}-4 t^{2}+1\right) t^{2}}{\left(9 t^{2}+2 t+1\right)^{2}\left(9 t^{2}-2 t+1\right)^{2}}$ \\
\hline $12 \mathrm{H}$ & $\frac{\left(54441 t^{6}+47142 t^{5}+14751 t^{4}+1908 t^{3}+87 t^{2}+6 t+1\right) t^{2}}{(5 t+1)^{2}(4 t+1)^{2}\left(39 t^{2}+6 t-1\right)^{2}}$ \\
\hline $12 I$ & $\frac{\left(3 t^{2}+1\right)^{4} t^{2}}{(t-1)^{2}(3 t-1)^{2}(3 t+1)^{2}(t+1)^{2}}$ \\
\hline $12 \mathrm{~J}$ & $\frac{\left(4096 t^{12}-1600 t^{6}+1\right) t^{2}}{\left(4 t^{2}+1\right)^{2}\left(16 t^{4}-4 t^{2}+1\right)^{2}}$ \\
\hline $13 \mathrm{~A}$ & $\frac{\left(41472 t^{6}+36048 t^{5}+11440 t^{4}+1512 t^{3}+73 t^{2}+6 t+1\right) t^{2}}{(4 t+1)^{2}(3 t+1)^{2}\left(48 t^{2}+4 t-1\right)^{2}}$ \\
\hline $13 B$ & $-\frac{\left(-1+447 t^{8}-3342 t^{7}-1345 t^{6}-1302 t^{5}-224 t^{4}-74 t^{3}-t^{2}-2 t\right)}{(2 t-1)^{2}\left(7 t^{2}+t+1\right)^{2}\left(5 t^{2}+2 t+1\right)^{2}}$ \\
\hline $14 \mathrm{~A}$ & $\frac{\left(17688 t^{6}+18888 t^{5}+7009 t^{4}+1036 t^{3}+62 t^{2}+4 t+1\right) t^{2}}{(2 t+1)^{2}(6 t+1)^{2}\left(23 t^{2}+6 t-1\right)^{2}}$ \\
\hline $14 B$ & $-\frac{\left(-1+1832 t^{8}-1544 t^{7}-2385 t^{6}+2802 t^{5}-1063 t^{4}+180 t^{3}-15 t^{2}+2 t\right) t^{2}}{(5 t+1)^{2}(3 t-1)^{2}(2 t-1)^{2}\left(2 t^{2}-t+1\right)^{2}}$ \\
\hline
\end{tabular}


Table A.1. (Continued.)

\begin{tabular}{|c|c|}
\hline Class & $R(f)$ \\
\hline \multirow{2}{*}{$14 C$} & $\left(285777 t^{8}+454332 t^{7}+293690 t^{6}+98208 t^{5}+17779 t^{4}+1696 t^{3}+106 t^{2}+12 t+1\right) t^{2}$ \\
\hline & $(4 t+1)^{2}\left(391 t^{4}+278 t^{3}+53 t^{2}-2 t-1\right)^{2}$ \\
\hline \multirow{2}{*}{$15 A$} & $\left(10540 t^{6}+15628 t^{5}+6841 t^{4}+1040 t^{3}+46 t^{2}+4 t+1\right) t^{2}$ \\
\hline & $(8 t-1)^{2}(4 t+1)^{2}\left(13 t^{2}+6 t+1\right)^{2}$ \\
\hline \multirow{2}{*}{$15 B$} & $-\frac{\left(-1+1175 t^{8}-120 t^{7}-1078 t^{6}-488 t^{5}+357 t^{4}-152 t^{3}+2 t^{2}\right) t^{2}}{-}$ \\
\hline & $(2 t-1)^{2}\left(25 t^{4}-10 t^{3}+11 t^{2}+2 t+1\right)^{2}$ \\
\hline \multirow{2}{*}{$15 C$} & $\left(125800 t^{8}+220760 t^{7}+158768 t^{6}+59408 t^{5}+12073 t^{4}+1282 t^{3}+83 t^{2}+10 t+1\right) t^{2}$ \\
\hline & $(3 t+1)^{2}\left(25 t^{2}+5 t-1\right)^{2}\left(11 t^{2}+7 t+1\right)^{2}$ \\
\hline \multirow{2}{*}{$15 D$} & $\left(15625 t^{12}-8000 t^{9}-1750 t^{6}-64 t^{3}+1\right) t^{2}$ \\
\hline & $\overline{\left(5 t^{2}-2 t+1\right)^{2}\left(25 t^{4}+10 t^{3}-t^{2}+2 t+1\right)^{2}}$ \\
\hline \multirow{2}{*}{$16 \mathrm{~A}$} & $\left(256 t^{8}+256 t^{6}+608 t^{4}+16 t^{2}+1\right) t^{2}$ \\
\hline & $\left(4 t^{2}+4 t-1\right)^{2}\left(4 t^{2}-4 t-1\right)^{2}$ \\
\hline \multirow{2}{*}{$16 B$} & $\left(16 t^{4}-16 t^{3}+8 t^{2}+4 t+1\right)\left(16 t^{4}+16 t^{3}+8 t^{2}-4 t+1\right) t^{2}$ \\
\hline & $(2 t-1)^{2}(2 t+1)^{2}\left(4 t^{2}+1\right)^{2}$ \\
\hline \multirow{2}{*}{$16 C$} & $\left(12864 t^{6}+12864 t^{5}+4912 t^{4}+800 t^{3}+44 t^{2}+4 t+1\right) t^{2}$ \\
\hline & $(4 t+1)^{2}(2 t+1)^{2}\left(28 t^{2}+4 t-1\right)^{2}$ \\
\hline \multirow{2}{*}{$17 A$} & $\left(41200 t^{8}+84016 t^{7}+70840 t^{6}+30984 t^{5}+7265 t^{4}+864 t^{3}+58 t^{2}+8 t+1\right) t^{2}$ \\
\hline & $(2 t+1)^{2}\left(212 t^{4}+176 t^{3}+39 t^{2}-2 t-1\right)^{2}$ \\
\hline \multirow{2}{*}{$18 \mathrm{~A}$} & $\left(2024 t^{8}+3936 t^{7}-176 t^{6}+896 t^{5}-156 t^{4}+40 t^{3}-4 t^{2}+1\right) t^{2}$ \\
\hline & $(t-1)^{2}\left(8 t^{2}+1\right)^{2}\left(7 t^{2}+t+1\right)^{2}$ \\
\hline \multirow{2}{*}{$18 B$} & $\left(3617 t^{6}+4978 t^{5}+2563 t^{4}+468 t^{3}+35 t^{2}+2 t+1\right) t^{2}$ \\
\hline & $(5 t+1)^{2}(2 t+1)^{2}(7 t-1)^{2}(t+1)^{2}$ \\
\hline \multirow{2}{*}{$18 C$} & $\left(1-2 t+6 t^{2}-50 t^{3}+85 t^{4}\right)^{2} t^{2}$ \\
\hline & $\overline{(3 t-1)^{2}(2 t-1)^{2}(t+1)^{2}\left(11 t^{2}-2 t-1\right)^{2}}$ \\
\hline \multirow{2}{*}{$18 D$} & $\left(2 t^{2}-2 t-1\right)^{2}\left(4 t^{4}+4 t^{3}+6 t^{2}-2 t+1\right)^{2} t^{2}$ \\
\hline & $\overline{(2 t-1)^{2}(t+1)^{2}\left(t^{2}-t+1\right)^{2}\left(4 t^{2}+2 t+1\right)^{2}}$ \\
\hline \multirow{2}{*}{$18 E$} & $\left(30472 t^{8}+68192 t^{7}+60688 t^{6}+27392 t^{5}+6532 t^{4}+776 t^{3}+52 t^{2}+8 t+1\right) t^{2}$ \\
\hline & $(3 t+1)^{2}\left(7 t^{2}+5 t+1\right)^{2}\left(20 t^{2}+4 t-1\right)^{2}$ \\
\hline \multirow{2}{*}{$19 \mathrm{~A}$} & $t^{2}\left(1+11340 t^{8}+29064 t^{7}+29212 t^{6}+14908 t^{5}+4120 t^{4}+580 t^{3}+41 t^{2}+6 t\right)$ \\
\hline & $2(4 t+1)^{2}(3 t+1)^{2}\left(12 t^{3}+16 t^{2}+4 t-1\right)^{2}$ \\
\hline \multirow{2}{*}{$20 \mathrm{~A}$} & $t^{2}\left(1+3072 t^{6}+2304 t^{5}+1088 t^{4}+304 t^{3}+32 t^{2}\right)$ \\
\hline & $(4 t+1)^{2}\left(16 t^{2}+4 t-1\right)^{2}$ \\
\hline \multirow{2}{*}{$20 B$} & $t^{2}\left(1+3072 t^{8}-704 t^{6}+848 t^{4}+8 t^{2}\right)$ \\
\hline & $(4 t-1)^{2}(4 t+1)^{2}\left(4 t^{2}+1\right)^{2}$ \\
\hline \multirow{2}{*}{$20 C$} & $t^{2}\left(t^{2}+1\right)\left(13 t^{2}-6 t+1\right)\left(61 t^{4}-62 t^{3}+14 t^{2}+2 t+1\right)$ \\
\hline & $(t-1)^{2}(2 t-1)^{2}(3 t+1)^{2}\left(5 t^{2}-2 t+1\right)^{2}$ \\
\hline \multirow{2}{*}{$20 D$} & $t^{2}\left(1+4096 t^{12}-1024 t^{8}-576 t^{6}-64 t^{4}\right)$ \\
\hline & $\left(4 t^{2}+1\right)^{2}\left(16 t^{4}+12 t^{2}+1\right)^{2}$ \\
\hline \multirow{2}{*}{$20 E$} & $t^{2}\left(1+t^{12}+10 t^{10}+351 t^{8}+44 t^{6}+351 t^{4}+10 t^{2}\right)$ \\
\hline & $(t-1)^{2}(t+1)\left(t^{2}+4 t^{2}-1\right)^{2}\left(t^{2}-4 t-1\right)^{2}$ \\
\hline
\end{tabular}


Table A.1. (Continued.)

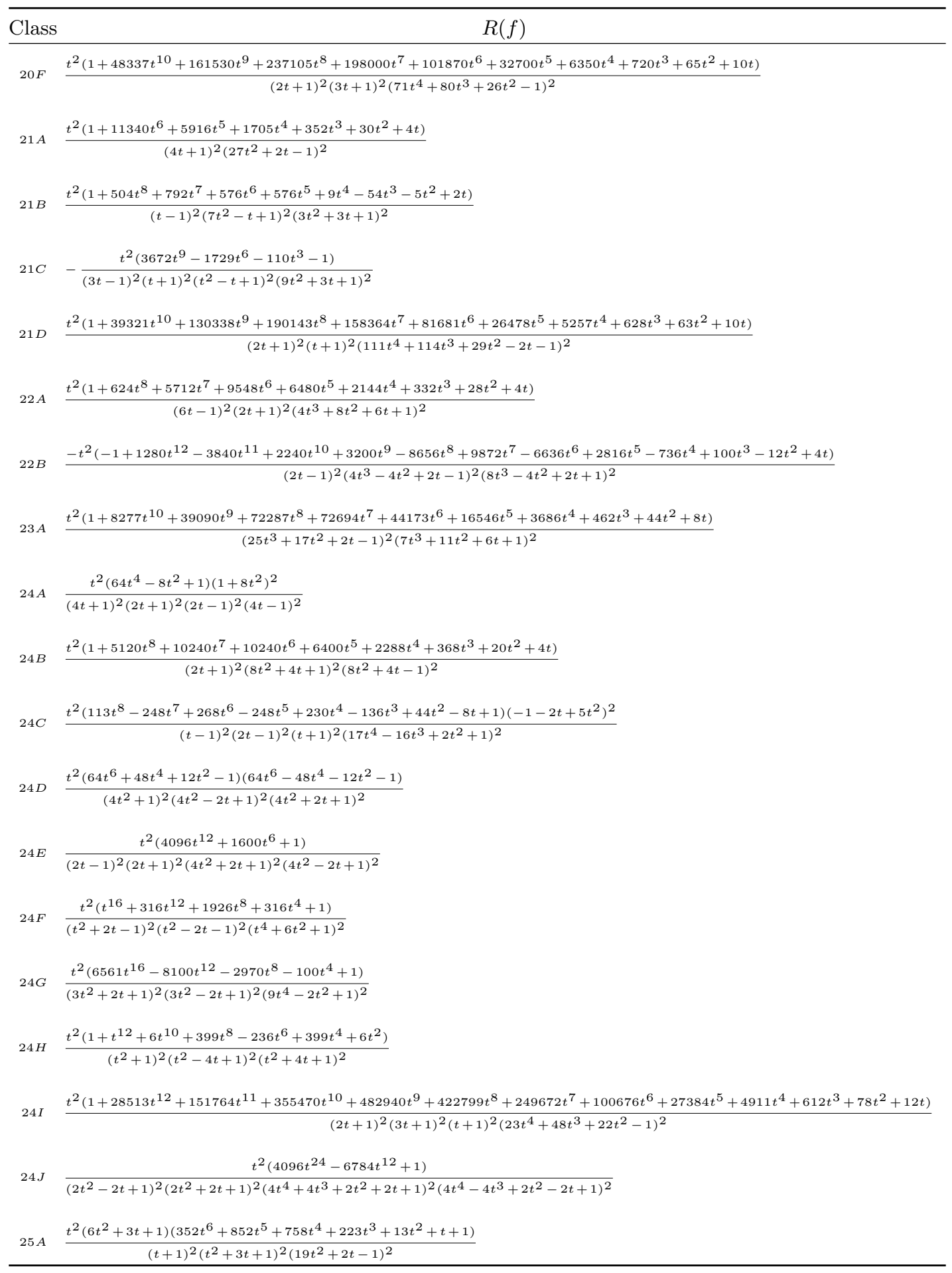


Table A.1. (Continued.)

\begin{tabular}{|c|c|}
\hline Class & $R(f)$ \\
\hline $26 \mathrm{~A}$ & $\frac{t^{2}\left(1+3840 t^{8}+7376 t^{7}+6624 t^{6}+3888 t^{5}+1345 t^{4}+228 t^{3}+22 t^{2}+4 t\right)}{(4 t+1)^{2}\left(16 t^{3}+15 t^{2}+2 t-1\right)^{2}}$ \\
\hline $26 B$ & $\begin{array}{l}\left(t^{2}\left(1+83097 t^{14}+455190 t^{13}+1192717 t^{12}+1963740 t^{11}+2241876 t^{10}+1851100 t^{9}+1122882 t^{8}+500052 t^{7}\right.\right. \\
\left.\left.+161298 t^{6}+36852 t^{5}+5908 t^{4}+740 t^{3}+101 t^{2}+14 t\right)\right) \\
/\left((2 t+1)^{2}(t+1)^{2}\left(191 t^{6}+384 t^{5}+308 t^{4}+114 t^{3}+12 t^{2}-4 t-1\right)^{2}\right)\end{array}$ \\
\hline $27 A ; 27 B$ & $\frac{t^{2}\left(1+5832 t^{9}+17496 t^{8}+23328 t^{7}+17577 t^{6}+7938 t^{5}+2079 t^{4}+288 t^{3}+27 t^{2}+6 t\right)}{(3 t+1)^{2}\left(9 t^{3}+9 t^{2}+3 t-1\right)^{2}\left(3 t^{2}+3 t+1\right)^{2}}$ \\
\hline $28 \mathrm{~A}$ & $\frac{t^{2}\left(1+31752 t^{10}-19583 t^{8}+3820 t^{6}-250 t^{4}+4 t^{2}\right)}{(2 t+1)^{2}(2 t-1)^{2}\left(7 t^{2}+2 t-1\right)^{2}\left(7 t^{2}-2 t-1\right)^{2}}$ \\
\hline $28 B$ & $\frac{-t^{2}\left(-1+1832 t^{8}+1544 t^{7}-2385 t^{6}-2802 t^{5}-1063 t^{4}-180 t^{3}-15 t^{2}-2 t\right)}{(2 t+1)^{2}(3 t+1)^{2}(5 t-1)^{2}\left(2 t^{2}+t+1\right)^{2}}$ \\
\hline $28 C$ & $\frac{t^{2}\left(3 t^{2}+1\right)\left(280 t^{12}+1715 t^{10}+1941 t^{8}+94 t^{6}+66 t^{4}-t^{2}+1\right)}{(t-1)^{2}(t+1)^{2}\left(7 t^{2}+1\right)^{2}\left(2 t^{2}-t+1\right)^{2}\left(2 t^{2}+t+1\right)^{2}}$ \\
\hline $28 D$ & $\frac{t^{2}\left(1+t^{16}+4 t^{14}+170 t^{12}+80 t^{10}-909 t^{8}+80 t^{6}+170 t^{4}+4 t^{2}\right)}{\left(t^{4}+2 t^{3}-5 t^{2}+2 t+1\right)^{2}\left(t^{4}-2 t^{3}-5 t^{2}-2 t+1\right)^{2}}$ \\
\hline $29 \mathrm{~A}$ & $\frac{t^{2}\left(1+624 t^{12}+7248 t^{11}+28472 t^{10}+53152 t^{9}+59625 t^{8}+45520 t^{7}+24526 t^{6}+9000 t^{5}+2091 t^{4}+296 t^{3}+38 t^{2}+8 t\right)}{(2 t+1)^{2}\left(4 t^{6}+32 t^{5}+83 t^{4}+66 t^{3}+17 t^{2}-2 t-1\right)^{2}}$ \\
\hline $30 A$ & $\frac{t^{2}\left(1+368536 t^{12}-708784 t^{11}+633016 t^{10}-318416 t^{9}+61972 t^{8}+23264 t^{7}-15664 t^{6}+2432 t^{5}+289 t^{4}-158 t^{3}+43 t^{2}-10 t\right)}{(3 t-1)^{2}\left(t^{2}-3 t+1\right)^{2}\left(11 t^{2}-t-1\right)^{2}\left(8 t^{2}-1\right)^{2}}$ \\
\hline $30 B$ & $\frac{t^{2}\left(1+2700 t^{8}-2340 t^{7}+2613 t^{6}+1386 t^{5}+311 t^{4}+112 t^{3}+15 t^{2}+2 t\right)}{(t-1)^{2}(3 t+1)^{2}(4 t+1)^{2}(5 t-1)^{2}}$ \\
\hline $30 C$ & $\frac{t^{2}\left(1+2700 t^{12}-5580 t^{11}+7077 t^{10}-2658 t^{9}+4169 t^{8}-3368 t^{7}+2710 t^{6}-1244 t^{5}+362 t^{4}-76 t^{3}+5 t^{2}-2 t\right)}{(t-1)^{2}(3 t+1)^{2}(4 t 2-t+1)^{2}\left(5 t^{2}-2 t+1\right)^{2}}$ \\
\hline $30 D$ & $\frac{t^{2}\left(1+46521 t^{12}+155646 t^{11}+231861 t^{10}+209214 t^{9}+135662 t^{8}+71734 t^{7}+31681 t^{6}+10642 t^{5}+2414 t^{4}+362 t^{3}+53 t^{2}+10 t\right)}{(2 t+1)^{2}\left(7 t^{2}+5 t+1\right)^{2}\left(9 t^{2}+3 t-1\right)^{2}\left(t^{2}-t-1\right)^{2}}$ \\
\hline $30 E$ & $\frac{t^{2}\left(t^{18}+70 t^{15}+291 t^{12}+1004 t^{9}+291 t^{6}+70 t^{3}+1\right)}{(t-1)^{2}\left(t^{2}+t+1\right)^{2}\left(t^{2}-3 t+1\right)^{2}\left(t^{4}+3 t^{3}+8 t^{2}+3 t+1\right)^{2}}$ \\
\hline $30 F$ & $\frac{-t^{2}\left(-1+40 t^{12}+240 t^{11}-1080 t^{10}-7600 t^{9}-17172 t^{8}-20448 t^{7}-14360 t^{6}-6072 t^{5}-1497 t^{4}-210 t^{3}-27 t^{2}-6 t\right)}{(t+1)^{2}\left(t^{2}+t+1\right)^{2}\left(5 t^{2}+5 t+1\right)^{2}\left(4 t^{2}+4 t-1\right)^{2}}$ \\
\hline $30 G$ & $\begin{array}{l}\left(t^{2}\left(1+1024 t^{20}-5120 t^{19}+12032 t^{18}-22784 t^{17}+54592 t^{16}-132736 t^{15}+238528 t^{14}-293696 t^{13}+249824 t^{12}\right.\right. \\
\left.\left.-159232 t^{11}+99968 t^{10}-79616 t^{9}+62456 t^{8}-36712 t^{7}+14908 t^{6}-4148 t^{5}+853 t^{4}-178 t^{3}+47 t^{2}-10 t\right)\right) \\
/\left((t-1)^{2}(2 t-1)^{2}(4 t 2-2 t-1)^{2}\left(t^{2}+t-1\right)^{2}\left(4 t^{4}-6 t^{3}+5 t^{2}-3 t+1\right)^{2}\right)\end{array}$ \\
\hline $31 A$ & $\frac{t^{2}\left(1+648 t^{12}+3864 t^{11}+10189 t^{10}+22122 t^{9}+35047 t^{8}+33086 t^{7}+18981 t^{6}+6930 t^{5}+1622 t^{4}+246 t^{3}+36 t^{2}+8 t\right)}{\left(27 t^{3}+17 t^{2}-1\right)^{2}\left(t^{3}+3 t^{2}+4 t+1\right)^{2}}$ \\
\hline $32 \mathrm{~A}$ & $\frac{-t^{2}\left(-1+2496 t^{12}-768 t^{11}-25664 t^{10}-60800 t^{9}-74096 t^{8}-56576 t^{7}-28640 t^{6}-9600 t^{5}-2044 t^{4}-272 t^{3}-36 t^{2}-8 t\right)}{(2 t+1)^{2}\left(2 t^{2}+2 t+1\right)^{2}\left(28 t^{4}+32 t^{3}+12 t^{2}-1\right)^{2}}$ \\
\hline $32 B$ & $\frac{t^{2}\left(16 t^{8}+56 t^{4}+1\right)\left(1+2 t^{2}\right)^{2}}{\left(2 t^{2}-1\right)^{2}\left(2 t^{2}+4 t+1\right)^{2}\left(2 t^{2}-4 t+1\right)^{2}}$ \\
\hline
\end{tabular}


Table A.1. (Continued.)

\begin{tabular}{|c|c|}
\hline Class & $R(f)$ \\
\hline $33 \mathrm{~A}$ & $\begin{array}{l}-t^{2}\left(-1+1584 t^{16}-4312 t^{15}+5368 t^{14}-10912 t^{13}+11295 t^{12}-13318 t^{11}+9919 t^{10}-9038 t^{9}+5586 t^{8}-3966 t^{7}\right. \\
\left.+1843 t^{6}-790 t^{5}+214 t^{4}-30 t^{3}-5 t^{2}+2 t\right) /\left((t-1)^{2}\left(3 t^{2}-t+1\right)^{2}\left(11 t^{6}-11 t^{5}+20 t^{4}-3 t^{3}+8 t^{2}+t+1\right)^{2}\right)\end{array}$ \\
\hline $33 B$ & $\frac{t^{2}\left(1+1452 t^{10}+5104 t^{9}+9200 t^{8}+8092 t^{7}+4976 t^{6}+2756 t^{5}+1081 t^{4}+188 t^{3}+14 t^{2}+4 t\right)}{\left(11 t^{2}+2 t-1\right)^{2}\left(4 t^{3}+8 t^{2}+4 t+1\right)^{2}}$ \\
\hline $34 \mathrm{~A}$ & $\frac{t^{2}\left(1+432 t^{10}-912 t^{9}-664 t^{8}+752 t^{7}+1417 t^{6}+898 t^{5}+411 t^{4}+100 t^{3}+11 t^{2}+2 t\right)}{(2 t+1)^{2}(t+1)^{2}\left(2 t^{2}-3 t-1\right)^{2}\left(2 t^{2}-5 t+1\right)^{2}}$ \\
\hline $35 \mathrm{~A}$ & $\frac{-t^{2}\left(-1+6900 t^{10}+3360 t^{9}-12112 t^{8}-15964 t^{7}-11632 t^{6}-4780 t^{5}-1273 t^{4}-180 t^{3}-14 t^{2}-4 t\right)}{(2 t+1)^{2}\left(5 t^{2}+2 t+1\right)^{2}\left(20 t^{3}+4 t^{2}+2 t-1\right)^{2}}$ \\
\hline $35 B$ & $\begin{array}{l}t^{2}\left(1+1224 t^{16}+9048 t^{15}+33272 t^{14}+83320 t^{14}+160073 t^{12}+243226 t^{11}+290017 t^{10}+267514 t^{9}+188542 t^{8}\right. \\
\left.+100130 t^{7}+39221 t^{6}+10970 t^{5}+2130 t^{4}+314 t^{3}+53 t^{2}+10 t\right) \\
/\left((t+1)^{2}\left(t^{2}+3 t+1\right)^{2}\left(19 t^{6}+51 t^{5}+62 t^{4}+39 t^{3}+10 t^{2}-t-1\right)^{2}\right)\end{array}$ \\
\hline $36 \mathrm{~A}$ & $\frac{t^{2}\left(1+2 t+6 t^{2}+50 t^{3}+85 t^{4}\right)^{2}}{(t-1)^{2}(2 t+1)^{2}(3 t+1)^{2}\left(11 t^{2}+2 t-1\right)^{2}}$ \\
\hline $36 B$ & $\frac{t^{2}\left(2 t^{2}+2 t-1\right)^{2}\left(4 t^{4}-4 t^{3}+6 t^{2}+2 t+1\right)^{2}}{(t-1)^{2}(2 t+1)^{2}\left(t^{2}+t+1\right)^{2}\left(4 t^{2}-2 t+1\right)^{2}}$ \\
\hline $36 C$ & $\frac{t^{2}\left(1+729 t^{12}+162 t^{10}+2511 t^{8}-612 t^{6}+279 t^{4}+2 t^{2}\right)}{(t-1)^{2}(3 t-1)^{2}(3 t+1)^{2}(t+1)^{2}\left(3 t^{2}+1\right)^{2}}$ \\
\hline $36 D$ & $\begin{array}{l}\left(t^{2}\left(1+5184 t^{16}+41472 t^{15}+155520 t^{14}+362880 t^{13}+591840 t^{12}+720576 t^{11}+682560 t^{10}+514080 t^{9}+308376 t^{8}\right.\right. \\
\left.\left.+144864 t^{7}+51696 t^{6} \cdot+13536 t^{5}+2580 t^{4}+408 t^{3}+72 t^{2}+12 t\right)\right) \\
/\left((2 t+1)^{2}(t+1)^{2}\left(3 t^{2}+3 t+1\right)^{2}\left(12 t^{4}+24 t^{3}+12 t^{2}-1\right)^{2}\right)\end{array}$ \\
\hline $38 \mathrm{~A}$ & $\frac{t^{2}\left(1+768 t^{12}+2464 t^{11}+3308 t^{10}+3328 t^{9}+3976 t^{8}+5260 t^{7}+4532 t^{6}+2440 t^{5}+769 t^{4}+112 t^{3}+14 t^{2}+4 t\right)}{\left(4 t^{3}+4 t^{2}+4 t+1\right)^{2}\left(8 t^{3}+7 t^{2}+2 t-1\right)^{2}}$ \\
\hline $39 A$ & $\frac{t^{2}\left(1+648 t^{10}-3048 t^{9}+5608 t^{8}-4104 t^{7}+161 t^{6}+792 t^{5}+42 t^{3}+8 t^{2}\right)}{(t-1)^{2}(3 t+1)^{2}\left(3 t^{2}-5 t+1\right)^{2}\left(t^{2}-3 t-1\right)^{2}}$ \\
\hline $39 B$ & $\frac{-t^{2}\left(4104 t^{15}+2519 t^{12}-828 t^{9}-1006 t^{6}-36 t^{3}-1\right)}{(t+1)^{2}\left(t^{2}-t+1\right)^{2}\left(3 t^{2}+t-1\right)^{2}\left(9 t^{4}-3 t^{3}+4 t^{2}+t+1\right)^{2}}$ \\
\hline $39 C$ & $\begin{array}{l}\left(-t^{2}\left(-1+180 t^{18}+1620 t^{17}+6435 t^{16}+14760 t^{15}+19146 t^{14}+4242 t^{13}-40861 t^{12}-106092 t^{11}-158719 t^{10}\right.\right. \\
\left.\left.-166144 t^{9}-126450 t^{8}-70044 t^{7}-27877 t^{6}-7836 t^{5}-1567 t^{4}-260 t^{3}-51 t^{2}-10 t\right)\right) \\
/\left((2 t+1)^{2}(t+1)^{2}\left(3 t^{4}+6 t^{3}+8 t^{2}+5 t+1\right)^{2}\left(t^{4}+2 t^{3}+4 t^{2}+3 t-1\right)^{2}\right)\end{array}$ \\
\hline $40 \mathrm{~A}$ & $\frac{-t^{2}\left(7424 t^{12}-3344 t^{8}-104 t^{4}-1\right)}{(2 t+1)^{2}(2 t-1)^{2}\left(4 t^{2}+1\right)^{2}\left(2 t^{2}+2 t+1\right)^{2}\left(2 t^{2}-2 t+1\right)^{2}}$ \\
\hline $40 B$ & $\frac{t^{2}\left(1+4096 t^{12}-1024 t^{8}+576 t^{6}-64 t^{4}\right)}{(2 t+1)^{2}(2 t-1)^{2}\left(4 t^{2}-2 t-1\right)^{2}\left(4 t^{2}+2 t-1\right)^{2}}$ \\
\hline $40 \mathrm{C}$ & $\frac{t^{2}\left(t^{16}+4 t^{14}+180 t^{12}+636 t^{10}+1174 t^{8}+636 t^{6}+180 t^{4}+4 t^{2}+1\right)(t-1)^{2}(t+1)^{2}}{\left(t^{2}+1\right)^{2}\left(t^{4}+2 t^{3}-2 t^{2}+2 t+1\right)^{2}\left(t^{4}-2 t^{3}-2 t^{2}-2 t+1\right)^{2}}$ \\
\hline $41 \mathrm{~A}$ & $\begin{array}{l}\left(t^{2}\left(1+192 t^{16}-624 t^{15}+1184 t^{14}+848 t^{13}-8839 t^{12}-8504 t^{11}+11872 t^{10}+28440 t^{9}+27584 t^{8}+18616 t^{7}\right.\right. \\
\left.\left.+9914 t^{6}+3880 t^{5}+992 t^{4}+168 t^{3}+32 t^{2}+8 t\right)\right) /\left(-1-4 t+8 t^{2}+66 t^{3}+120 t^{4}+56 t^{5}-53 t^{6}-36 t^{7}+16 t^{8}\right)^{2}\end{array}$ \\
\hline
\end{tabular}


Table A.1. (Continued.)

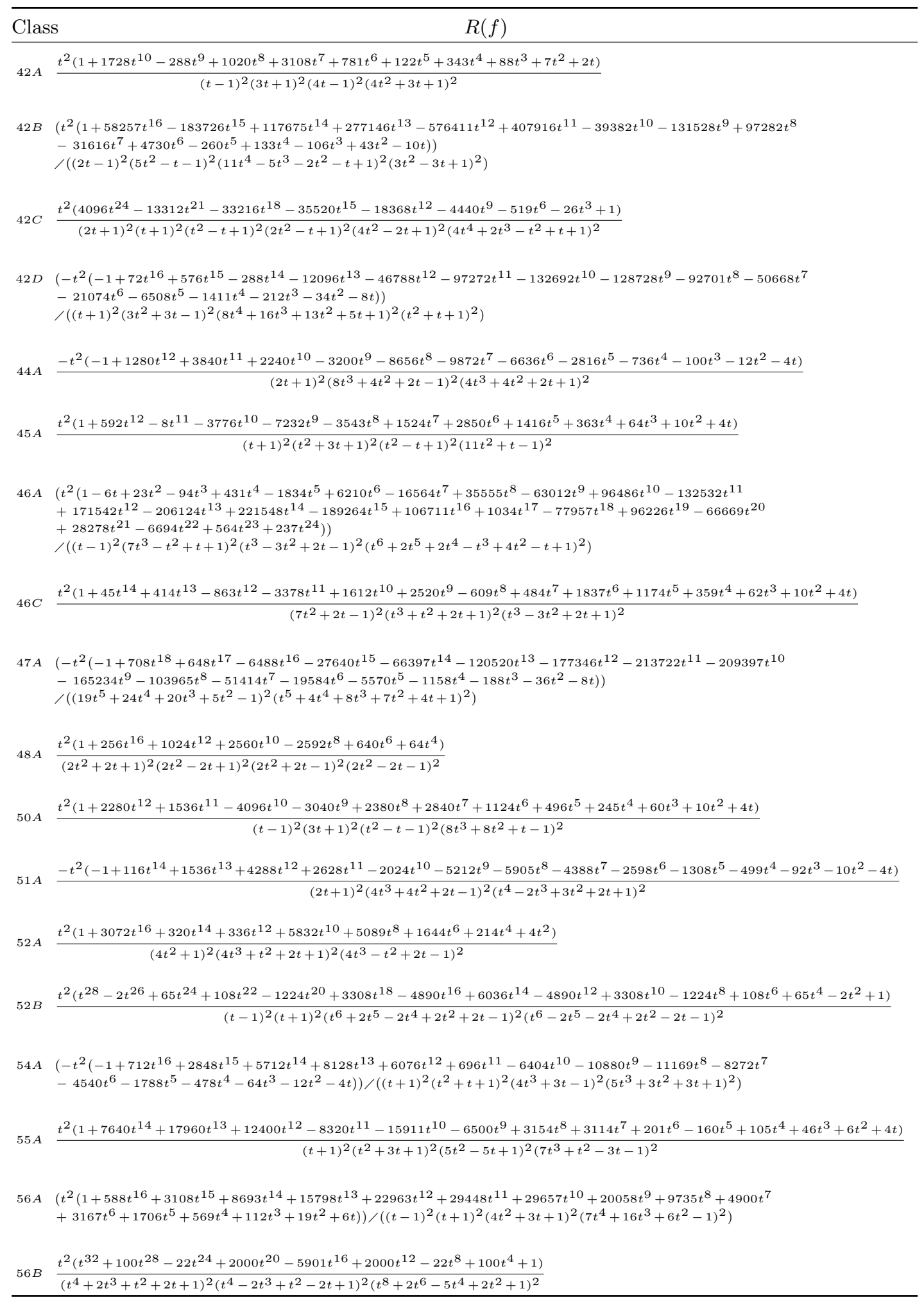


Table A.1. (Continued.)

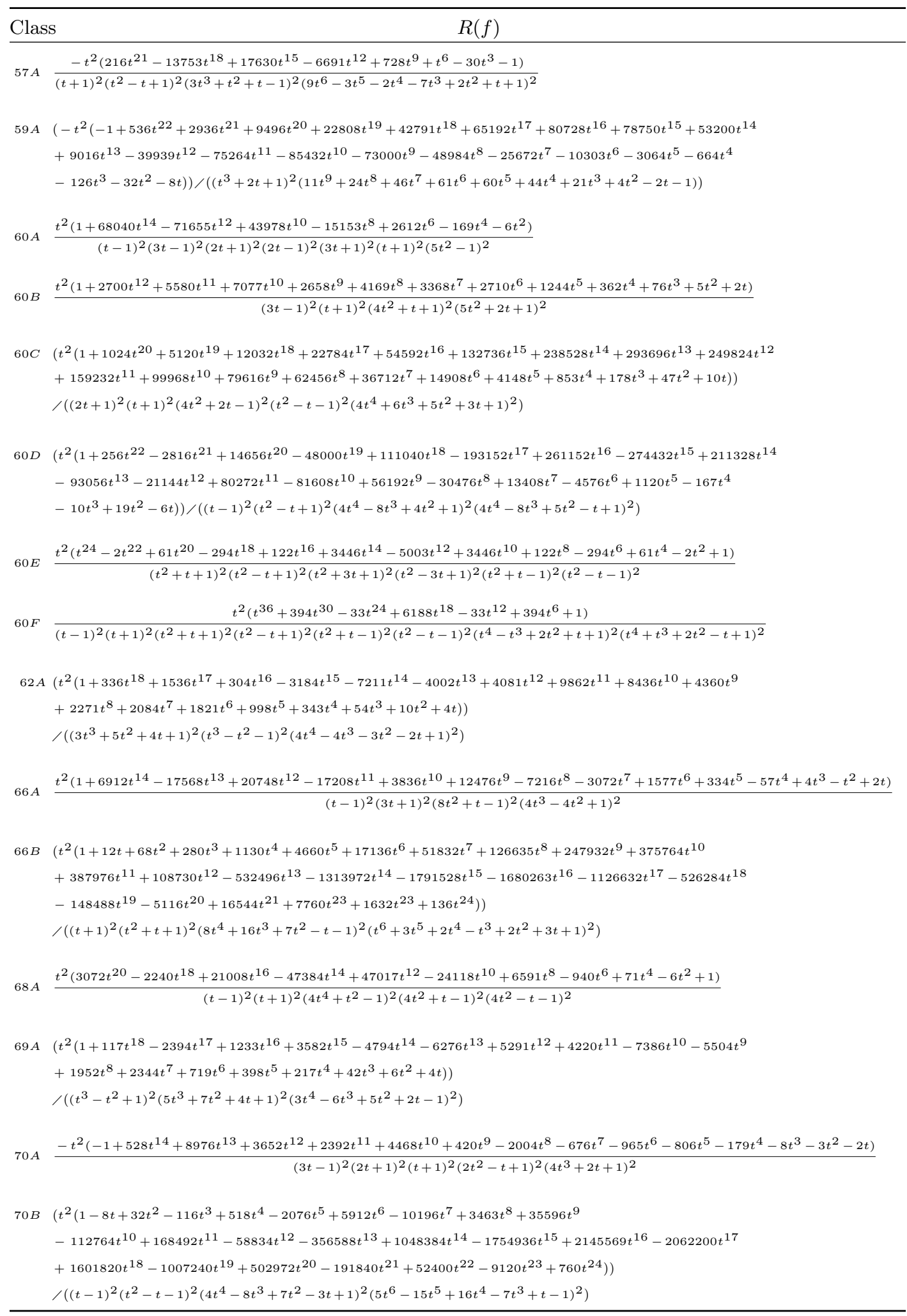


Table A.1. (Continued.)

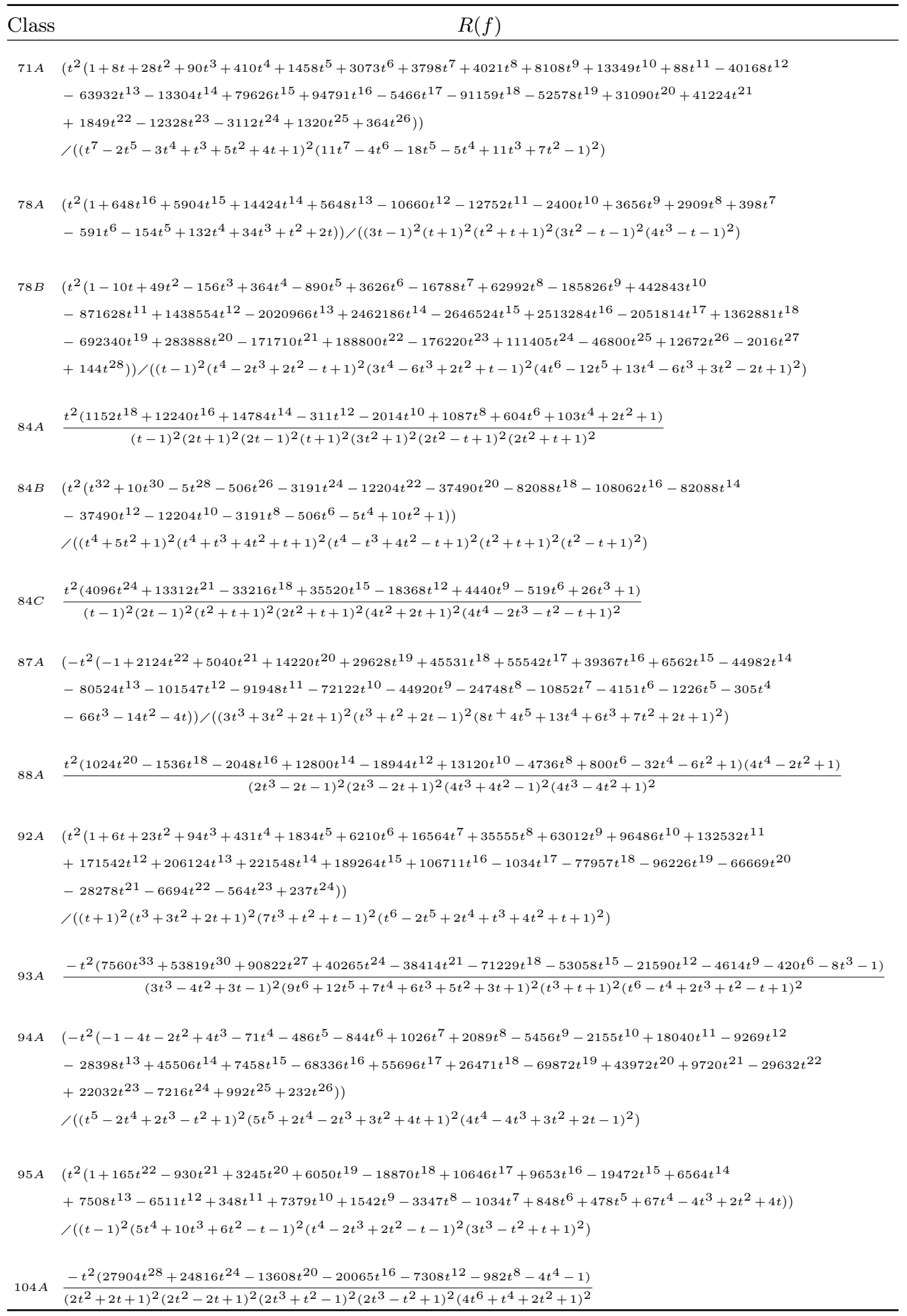


Table A.1. (Continued.)

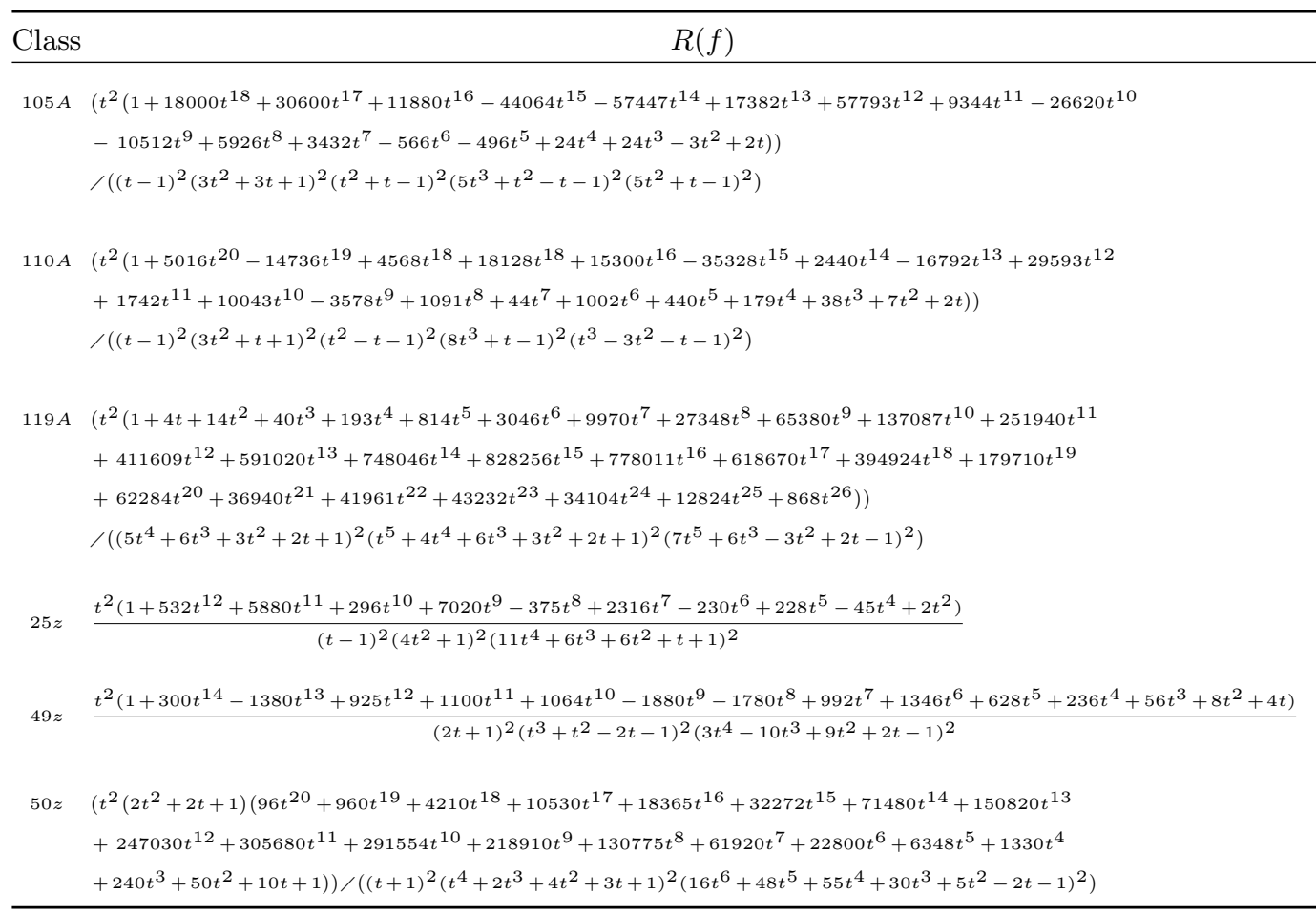

TABLE A.2. $R(f)$ for the remaining completely replicable functions.

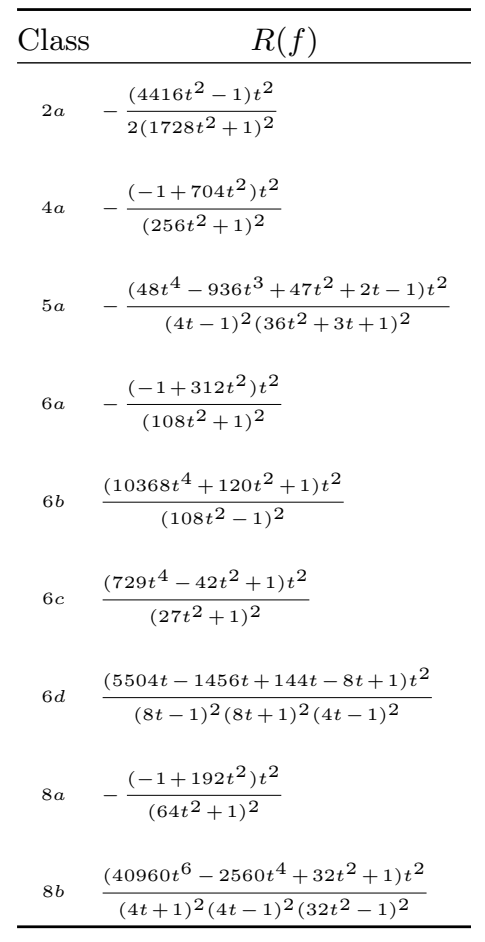


Table A.2. (Continued.)

\begin{tabular}{|c|c|}
\hline Class & $R(f)$ \\
\hline $8 c$ & $-\frac{\left(40960 t^{6}+2560 t^{4}+32 t^{2}-1\right) t^{2}}{2\left(32 t^{2}+1\right)^{2}\left(16 t^{2}+1\right)^{2}}$ \\
\hline $9 a$ & $\frac{\left(8748 t^{6}+540 t^{3}+1\right) t^{2}}{\left(108 t^{3}-1\right)^{2}}$ \\
\hline $9 b$ & $\frac{\left(1944 t^{4}-648 t^{3}+81 t^{2}-6 t+1\right) t^{2}}{(6 t-1)^{2}(3 t-1)^{2}(6 t+1)^{2}}$ \\
\hline $9 c$ & $\frac{\left(729 t^{6}-162 t^{3}+1\right) t^{2}}{(3 t+1)^{2}\left(9 t^{2}-3 t+1\right)^{2}}$ \\
\hline $9 d$ & $\frac{\left(3888 t^{8}+19440 t^{7}-648 t^{6}+2808 t^{5}-351 t^{4}+72 t^{3}-6 t^{2}+1\right) t^{2}}{\left(12 t^{2}+1\right)^{2}\left(18 t^{3}-9 t^{2}-1\right)^{2}}$ \\
\hline $10 a$ & $-\frac{\left(-1+1344 t^{6}-2288 t^{4}-40 t^{2}\right) t^{2}}{\left(-1+44 t^{2}+16 t^{4}\right)^{2}}$ \\
\hline $10 b$ & $\frac{\left(80 t^{6}-1896 t^{5}+1553 t^{4}-236 t^{3}+22 t^{2}-4 t+1\right) t^{2}}{(4 t+1)^{2}\left(28 t^{3}-17 t^{2}+6 t-1\right)^{2}}$ \\
\hline $10 c$ & $-\frac{\left(-1+1344 t^{6}+647 t^{4}+10 t^{2}\right) t^{2}}{\left(1+11 t^{2}+64 t^{4}\right)^{2}}$ \\
\hline $12 a$ & $-\frac{\left(-1+124416 t^{6}+6144 t^{4}+40 t^{2}\right) t^{2}}{\left(32 t^{2}+1\right)^{2}\left(36 t^{2}+1\right)^{2}}$ \\
\hline $12 b$ & $-\frac{\left(-1+6656 t^{6}-2304 t^{4}-24 t^{2}\right) t^{2}}{\left(4 t^{2}+1\right)^{2}\left(32 t^{2}-1\right)^{2}}$ \\
\hline $12 c$ & $\frac{\left(9216 t^{6}-1152 t^{4}+24 t^{2}+1\right) t^{2}}{(4 t+1)^{2}(4 t-1)^{2}\left(12 t^{2}-1\right)^{2}}$ \\
\hline $12 d$ & $\frac{\left(64 t^{4}+8 t^{2}+1\right)\left(-1+8 t^{2}\right)^{2}}{\left(1+4 t^{2}\right)^{2}\left(16 t^{2}+1\right)^{2}}$ \\
\hline $12 e$ & $\frac{\left(27648 t^{8}+2880 t^{6}-624 t^{4}+8 t^{2}+1\right) t^{2}}{(4 t+1)^{2}(4 t-1)^{2}\left(12 t^{2}-1\right)^{2}}$ \\
\hline $12 f$ & $-\frac{\left(-1+2240 t^{6}+240 t^{4}+24 t^{2}\right) t^{2}}{\left(1+4 t^{2}\right)^{2}\left(16 t^{2}+1\right)^{2}}$ \\
\hline $14 a$ & $-\frac{\left(-1+67704 t^{6}+4151 t^{4}+34 t^{2}\right) t^{2}}{\left(27 t^{2}+1\right)^{2}\left(28 t^{2}+1\right)^{2}}$ \\
\hline $14 b$ & $\frac{\left(2401 t^{8}-294 t^{6}-245 t^{4}-6 t^{2}+1\right) t^{2}}{\left(7 t^{2}+t+1\right)^{2}\left(7 t^{2}-t+1\right)^{2}}$ \\
\hline $14 c$ & $\frac{\left(1848 t^{6}+721 t^{4}+22 t^{2}+1\right) t^{2}}{(t-1)^{2}(t+1)^{2}\left(28 t^{2}-1\right)^{2}}$ \\
\hline $15 a$ & $\frac{\left(69040 t^{8}-58800 t^{7}+7000 t^{6}+4880 t^{5}-975 t^{4}-64 t^{3}+10 t^{2}+1\right) t^{2}}{(2 t-1)^{2}\left(11 t^{2}-2 t-1\right)^{2}\left(20 t^{2}-1\right)^{2}}$ \\
\hline $15 b$ & $\frac{\left(19980 t^{8}+16488 t^{7}+1500 t^{6}-2484 t^{5}-488 t^{4}+92 t^{3}+9 t^{2}+2 t+1\right) t^{2}}{(3 t-1)^{2}(2 t+1)^{2}\left(36 t^{3}+12 t^{2}-2 t-1\right)^{2}}$ \\
\hline $16 a$ & $\frac{\left(4096 t^{8}+640 t^{4}+1\right) t^{2}}{\left(8 t^{2}-1\right)^{2}\left(8 t^{2}+1\right)^{2}}$ \\
\hline
\end{tabular}


Table A.2. (Continued.)

\begin{tabular}{|c|c|}
\hline Class & $R(f)$ \\
\hline $16 b$ & $\frac{\left(6144 t^{6}-704 t^{4}+16 t^{2}+1\right) t^{2}}{(4 t+1)^{2}(4 t-1)^{2}\left(8 t^{2}-1\right)^{2}}$ \\
\hline $16 d$ & $\frac{\left(16 t^{4}-16 t^{2}+1\right)\left(16 t^{4}+16 t^{2}+1\right) t^{2}}{\left(16 t^{4}+1\right)^{2}}$ \\
\hline $16 e$ & $\frac{\left(32768 t^{10}-25600 t^{8}+5632 t^{6}-384 t^{4}+1\right) t^{2}}{\left(8 t^{2}-1\right)^{2}\left(32 t^{4}-8 t^{2}+1\right)^{2}}$ \\
\hline $16 f$ & $-\frac{\left(32768 t^{10}+25600 t^{8}+5632 t^{6}-384 t^{4}-1\right) t^{2}}{\left(8 t^{2}+1\right)^{2}\left(32 t^{4}+8 t^{2}+1\right)^{2}}$ \\
\hline $16 g$ & $-\frac{\left(-1+12288 t^{10}-22784 t^{8}+3328 t^{6}-32 t^{4}\right) t^{2}}{\left(8 t^{2}-1\right)^{2}\left(16 t^{4}+8 t^{2}-1\right)^{2}}$ \\
\hline $16 h$ & $\frac{\left(12288 t^{10}+22784 t^{8}+3328 t^{6}+32 t^{4}+1\right) t^{2}}{\left(8 t^{2}+1\right)^{2}\left(16 t^{4}-8 t^{2}-1\right)^{2}}$ \\
\hline $18 a$ & $\frac{\left(7520 t^{8}+4224 t^{7}+1660 t^{6}-1852 t^{5}-39 t^{4}+148 t^{3}+2 t^{2}+1\right) t^{2}}{(2 t+1)^{2}(2 t-1)^{2}\left(34 t^{3}+3 t^{2}-1\right)^{2}}$ \\
\hline $18 b$ & $\frac{\left(2808 t^{6}+1\right) t^{2}}{\left(108 t^{6}-1\right)^{2}}$ \\
\hline $18 c$ & $\frac{\left(729 t^{8}-324 t^{6}+702 t^{4}+12 t^{2}+1\right) t^{2}}{\left(-1+18 t^{2}+27 t^{4}\right)^{2}}$ \\
\hline $18 d$ & $\frac{\left(t^{12}+148 t^{9}+1590 t^{6}+148 t^{3}+1\right) t^{2}}{\left(t^{6}-34 t^{3}+1\right)}$ \\
\hline $18 e$ & $\frac{\left(4096 t^{12}-4352 t^{9}-660 t^{6}-68 t^{3}+1\right) t^{2}}{\left(4 t^{3}+1\right)^{2}\left(16 t^{3}+1\right)^{2}}$ \\
\hline $18 f$ & $-\frac{\left(-1+1080 t^{6}+135 t^{4}+18 t^{2}\right) t^{2}}{\left(12 t^{2}+1\right)^{2}\left(3 t^{2}+1\right)^{2}}$ \\
\hline $18 g$ & $\frac{\left(10368 t^{8}+1080 t^{6}-351 t^{4}+6 t^{2}+1\right) t^{2}}{(3 t-1)^{2}(3 t+1)^{2}\left(12 t^{2}-1\right)^{2}}$ \\
\hline $18 \mathrm{~h}$ & $\frac{\left(31808 t^{8}-36096 t^{7}+3820 t^{6}+3824 t^{5}-624 t^{4}-68 t^{3}+8 t^{2}+1\right) t^{2}}{(4 t-1)^{2}(4 t+1)^{2}\left(20 t^{3}-12 t^{2}+1\right)^{2}}$ \\
\hline $18 i$ & $\frac{\left(729 t^{12}-810 t^{6}+1\right) t^{2}}{\left(3 t^{2}+1\right)^{2}\left(3 t^{2}+3 t+1\right)^{2}\left(3 t^{2}-3 t+1\right)^{2}}$ \\
\hline $18 j$ & $\frac{t^{2}\left(1+1136 t^{8}-1968 t^{7}+376 t^{6}+896 t^{5}-39 t^{4}-80 t^{3}+2 t^{2}\right)}{(2 t+1)^{2}(2 t-1)^{2}\left(14 t s-3 t^{2}+1\right)^{2}}$ \\
\hline $20 a$ & $\frac{-t^{2}\left(-1+20160 t^{6}+1840 t^{4}+24 t^{2}\right)}{\left(16 t^{2}+1\right)^{2}\left(20 t^{2}+1\right)^{2}}$ \\
\hline $20 b$ & $\frac{t^{2}\left(1+4160 t^{6}-160 t^{4}+16 t^{2}\right)}{(2 t+1)^{2}(2 t-1)^{2}\left(20 t^{2}-1\right)^{2}}$ \\
\hline $20 c$ & $\frac{t^{2}\left(1+625 t^{8}-100 t^{6}-250 t^{4}-4 t^{2}\right)}{\left(5 t^{2}-2 t+1\right)^{2}\left(5 t^{2}+2 t+1\right)^{2}}$ \\
\hline $20 d$ & $\frac{t^{2}\left(3072 t^{10}+1280 t^{8}-1600 t^{6}+320 t^{4}+1\right)}{(2 t+1)^{2}(2 t-1)^{2}\left(16 t^{4}-4 t^{2}-1\right)^{2}}$ \\
\hline
\end{tabular}


Table A.2. (Continued.)

\begin{tabular}{|c|c|}
\hline Class & $R(f)$ \\
\hline $20 e$ & $\frac{t^{2}\left(1+49152 t^{12}+3392 t^{10}-14367 t^{8}+3708 t^{6}-282 t^{4}-4 t^{2}\right)}{\left(16 t^{3}+7 t^{2}-2 t-1\right)^{2}\left(16 t^{3}-7 t^{2}-2 t+1\right)^{2}}$ \\
\hline $22 a$ & $\frac{t^{2}\left(1+3520 t^{10}-4224 t^{8}+952 t^{6}+112 t^{4}+8 t^{2}\right)}{\left(-1+20 t^{2}-56 t^{4}+44 t^{6}\right)^{2}}$ \\
\hline $24 a$ & $\frac{-t^{2}\left(-1+9216 t^{6}+1152 t^{4}+24 t^{2}\right)}{\left(12 t^{2}+1\right)^{2}\left(16 t^{2}+1\right)^{2}}$ \\
\hline $24 b$ & $\frac{-t^{2}\left(-1+1152 t^{6}-720 t^{4}\right)}{\left(4 t^{2}+1\right)^{2}\left(12 t^{2}-1\right)^{2}}$ \\
\hline $24 c$ & $\frac{t^{2}\left(3 t^{2}-1\right)^{2}}{\left(9 t^{2}+1\right)^{2}\left(1+t^{2}\right)^{2}}$ \\
\hline $24 d$ & $\frac{-t^{2}\left(-1+2048 t^{10}-10752 t^{8}-3200 t^{6}-272 t^{4}\right)}{(2 t+1)^{2}(2 t-1)^{2}\left(4 t^{2}+1\right)^{2}\left(8 t^{2}+1\right)^{2}}$ \\
\hline $24 e$ & $\frac{t^{2}\left(1+2048 t^{10}+10752 t^{8}-3200 t^{6}+272 t^{4}\right)}{(2 t+1)^{2}(2 t-1)^{2}\left(4 t^{2}+1\right)^{2}\left(8 t^{2}-1\right)^{2}}$ \\
\hline $24 f$ & $\frac{-t^{2}\left(-1+92160 t^{10}+41472 t^{8}+7296 t^{6}+496 t^{4}\right)}{\left(4 t^{2}+1\right)^{2}\left(12 t^{2}+1\right)^{2}\left(8 t^{2}+1\right)^{2}}$ \\
\hline $24 \mathrm{~g}$ & $\frac{t^{2}\left(1+92160 t^{10}-41472 t^{8}+7296 t^{6}-496 t^{4}\right)}{(2 t+1)^{2}(2 t-1)^{2}\left(8 t^{2}-1\right)^{2}\left(12 t^{2}-1\right)^{2}}$ \\
\hline $24 h$ & $\frac{t^{2}\left(64 t^{6}-48 t^{4}+12 t^{2}+1\right)\left(64 t^{6}+48 t^{4}-12 t^{2}+1\right)}{(2 t+1)^{2}(2 t-1)^{2}\left(16 t^{4}-4 t^{2}+1\right)^{2}}$ \\
\hline $24 i$ & $\frac{t^{2}\left(1+59392 t^{14}-25088 t^{12}+25728 t^{10}-12656 t^{8}+2560 t^{6}-168 t^{4}-8 t^{2}\right)}{(2 t+1)^{2}(2 t-1)^{2}\left(8 t^{2}-1\right)^{2}\left(2 t^{2}+2 t-1\right)^{2}\left(2 t^{2}-2 t-1\right)^{2}}$ \\
\hline $24 j$ & $\frac{-t^{2}\left(-1+59392 t^{14}+25088 t^{12}+25728 t^{10}+12656 t^{8}+2560 t^{6}+168 t^{4}-8 t^{2}\right)}{\left(4 t^{2}+1\right)^{2}\left(8 t^{2}+1\right)^{2}\left(4 t^{4}+8 t^{2}+1\right)^{2}}$ \\
\hline $26 a$ & $\frac{t^{2}\left(1+192 t^{10}+2416 t^{8}-312 t^{6}+289 t^{4}+6 t^{2}\right)}{(t-1)^{2}(t+1)^{2}\left(16 t^{4}+12 t^{2}-1\right)^{2}}$ \\
\hline $27 a$ & $\frac{t^{2}\left(1+10692 t^{8}-9720 t^{7}+1296 t^{6}+1404 t^{5}-351 t^{4}-36 t^{3}+6 t^{2}\right)}{\left(12 t^{2}-1\right)^{2}\left(9 t^{3}-9 t^{2}+1\right)^{2}}$ \\
\hline $27 b$ & $\frac{t^{2}\left(-1-36 t^{3}+27 t^{6}\right)^{2}}{\left(-1+18 t^{3}+27 t^{6}\right)^{2}}$ \\
\hline $27 c$ & $\frac{t^{2}\left(729 t^{12}-972 t^{9}-594 t^{6}-36 t^{3}+1\right)}{\left(1+9 t^{3}+27 t^{6}\right)^{2}}$ \\
\hline $27 d$ & $\frac{t^{2}\left(8748 t^{12}+8100 t^{9}-1611 t^{6}+66 t^{3}+1\right)}{\left(12 t^{3}-1\right)^{2}\left(9 t^{3}-1\right)^{2}}$ \\
\hline $27 e$ & $\frac{t^{2}\left(648 t^{9}+873 t^{6}-42 t^{3}+1\right)}{\left(9 t^{3}+1\right)^{2}\left(3 t^{3}-1\right)^{2}}$ \\
\hline $28 a$ & $\frac{-t^{2}\left(-1+2184 t^{10}-1169 t^{8}-636 t^{6}-502 t^{4}-4 t^{2}\right)}{\left(4 t^{2}+1\right)^{2}\left(7 t^{4}+10 t^{2}-1\right)^{2}}$ \\
\hline $30 a$ & $\frac{-t^{2}\left(-1+711000 t^{10}+234423 t^{8}+27108 t^{6}+1138 t^{4}-4 t^{2}\right)}{\left(12 t^{2}+1\right)^{2}\left(125 t^{4}+22 t^{2}+1\right)^{2}}$ \\
\hline
\end{tabular}


Table A.2. (Continued.)

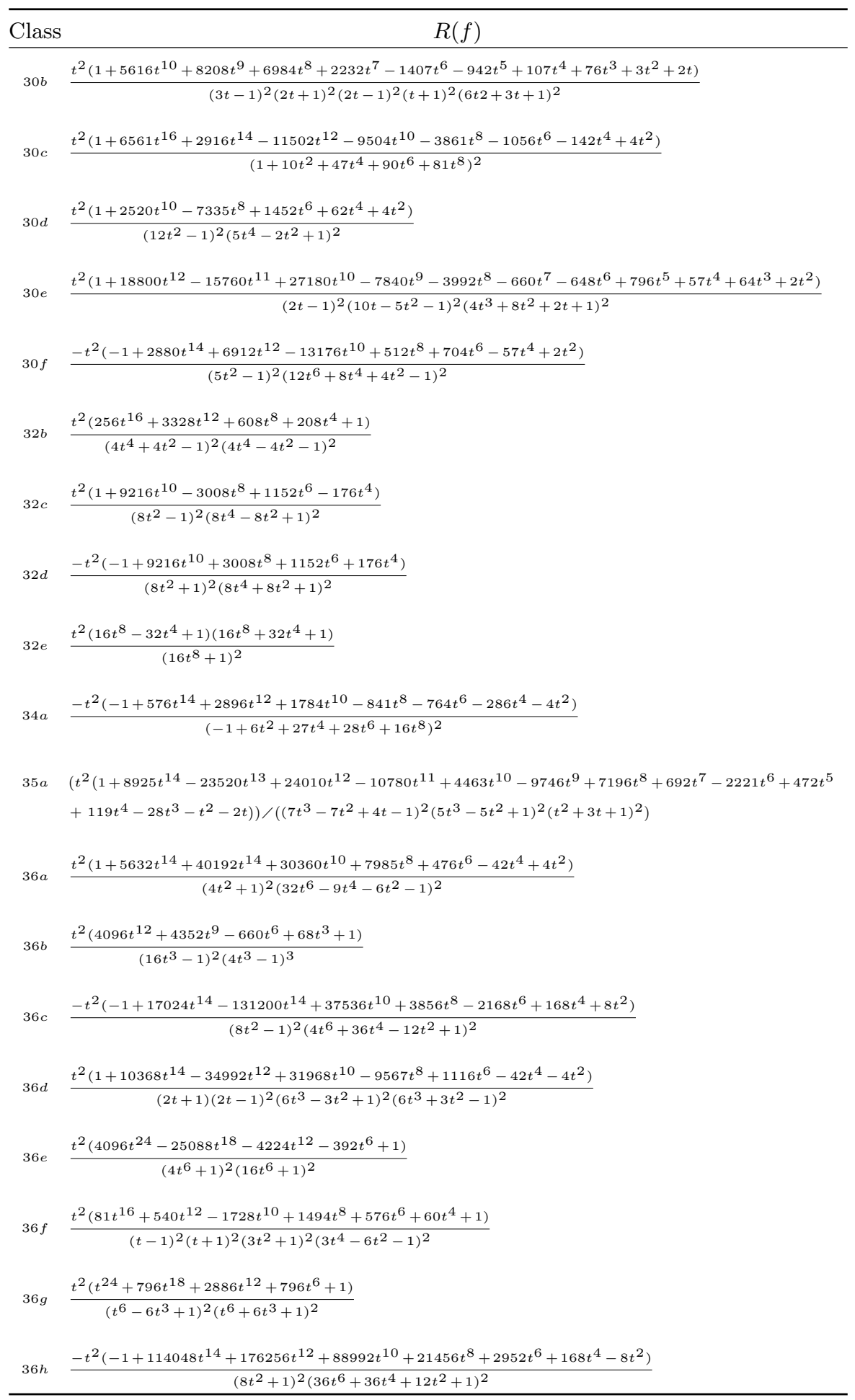


Table A.2. (Continued.)

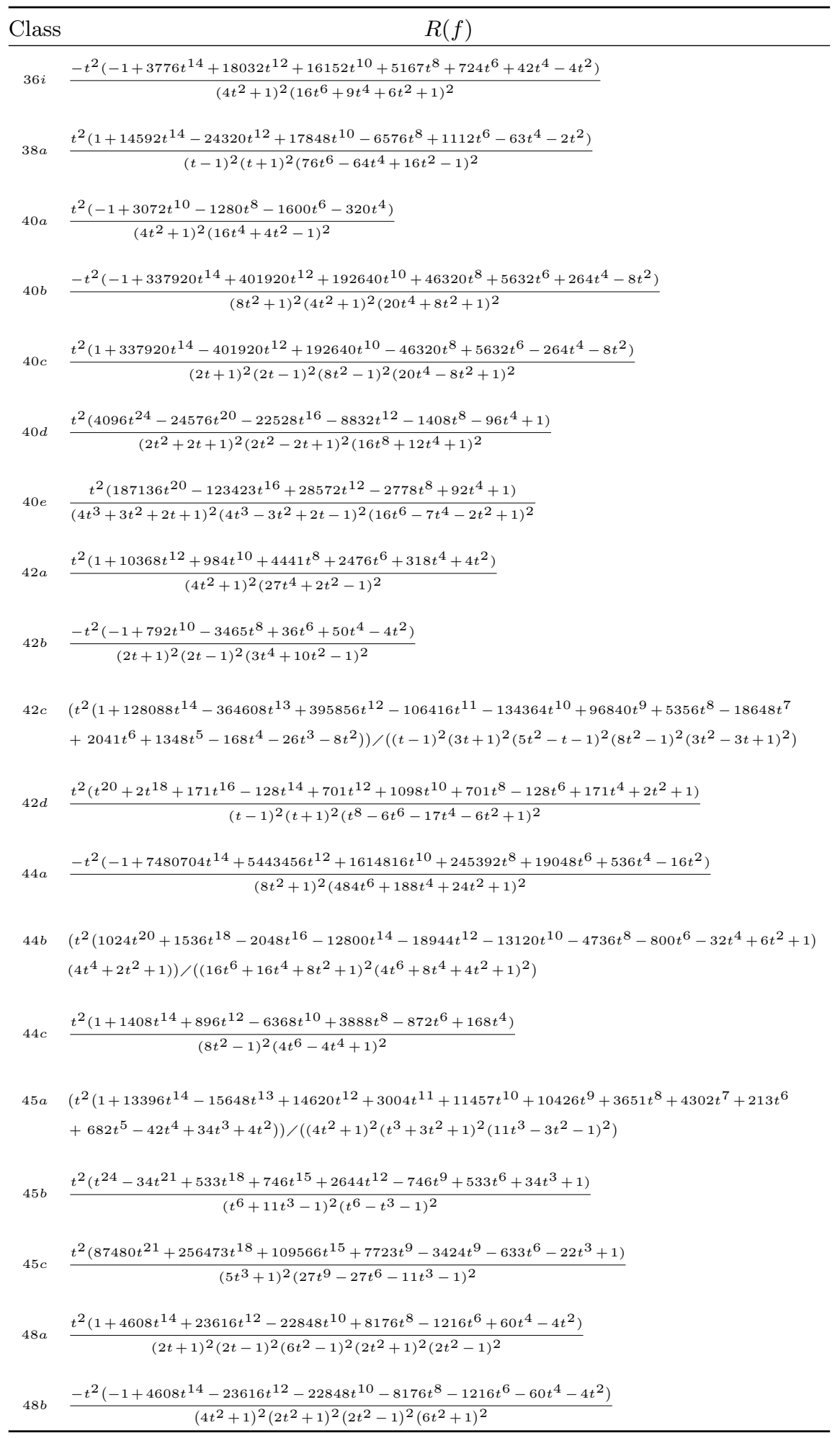


Table A.2. (Continued.)

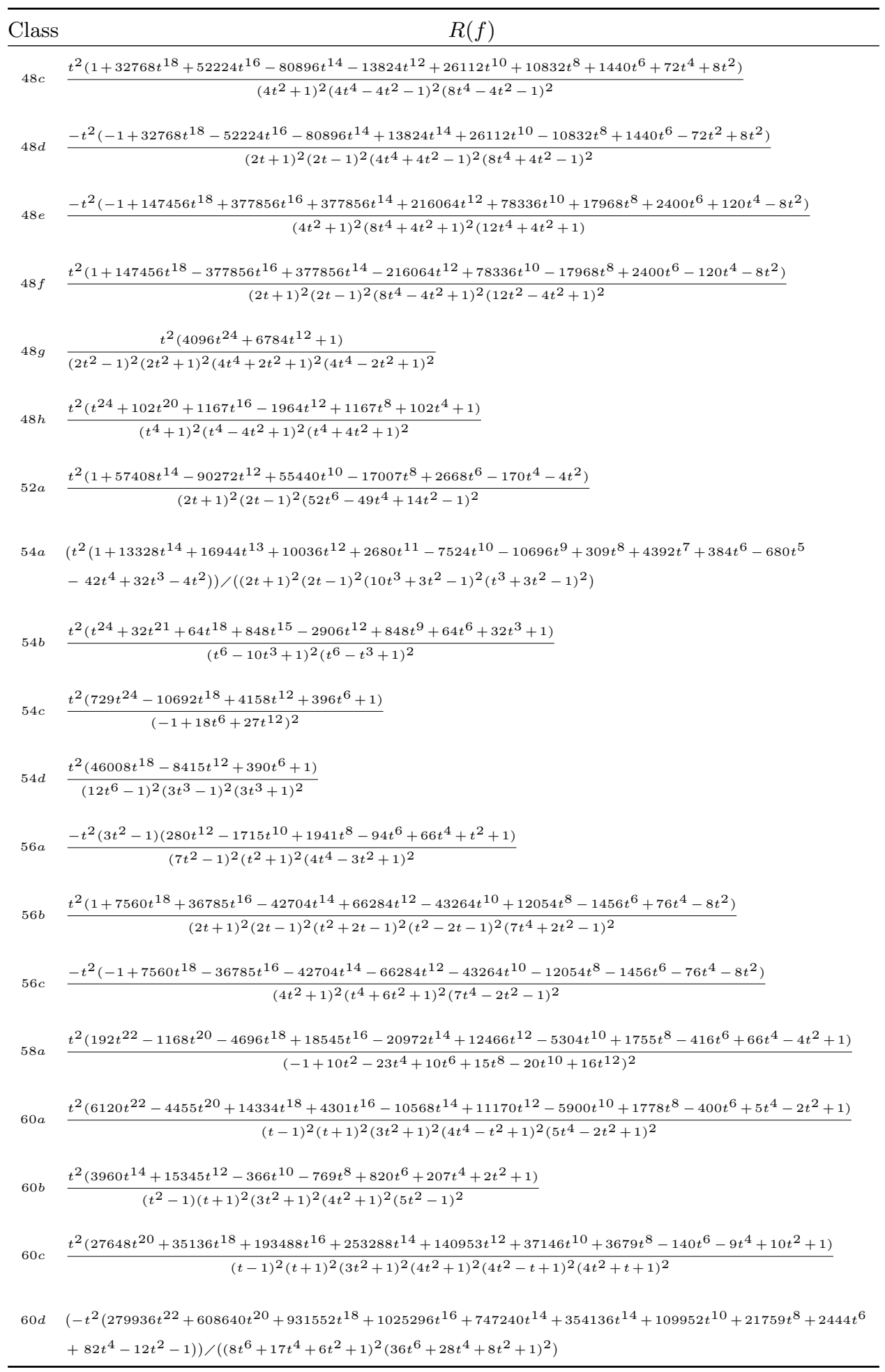


Table A.2. (Continued.)

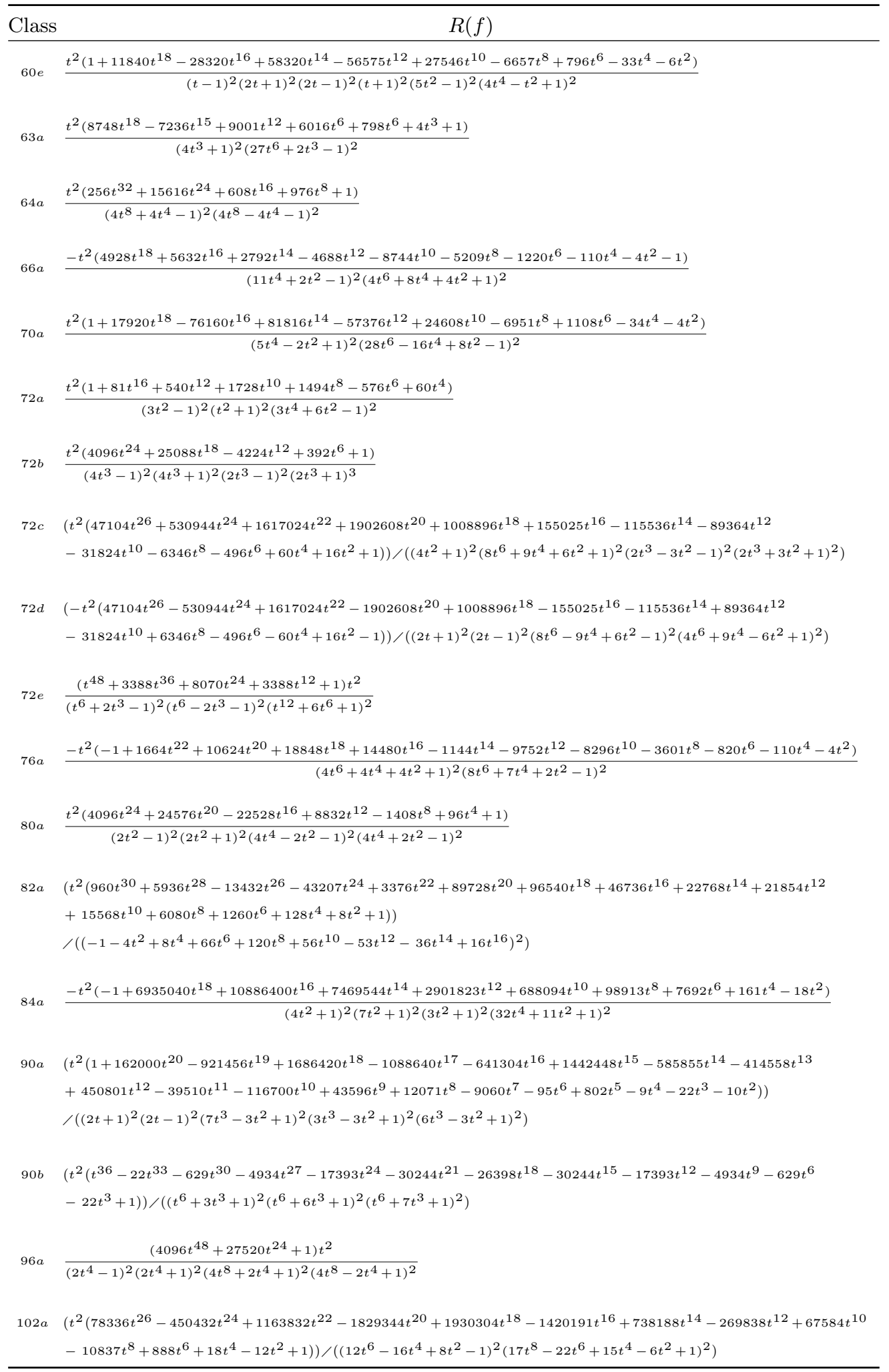


Table A.2. (Continued.)

\begin{tabular}{ll} 
Class & $R(f)$ \\
\hline $117 a$ & $\frac{t^{2}\left(8748 t^{30}-136620 t^{27}+751213 t^{24}-642942 t^{21}+139865 t^{18}+52758 t^{15}-34680 t^{12}+7506 t^{9}-715 t^{6}+18 t^{3}+1\right)}{\left(4 t^{3}-1\right)^{2}\left(27 t^{6}-11 t^{3}+1\right)^{2}\left(t^{6}+3 t^{3}-1\right)^{2}}$ \\
$120 a$ & $\frac{-t^{2}\left(6120 t^{22}+4455 t^{20}+14334 t^{18}-4301 t^{16}-10568 t^{14}-11170 t^{12}-5900 t^{10}-1778 t^{8}-400 t^{6}-5 t^{4}-2 t^{2}-1\right)}{\left(3 t^{2}-1\right)^{2}\left(t^{2}+1\right)^{2}\left(4 t^{4}+t^{2}+1\right)^{2}\left(5 t^{4}+2 t^{2}+1\right)^{2}}$ \\
$126 a$ & $\frac{-t^{2}\left(51624 t^{30}-33625 t^{24}-25132 t^{18}-3390 t^{12}-4 t^{6}-1\right)}{\left(4 t^{6}+1\right)^{2}\left(27 t^{12}+2 t^{6}-1\right)^{2}}$ \\
$132 a$ & $\left(t^{2}\left(14976 t^{26}+17280 t^{24}-114912 t^{22}+93872 t^{20}+48152 t^{18}-63736 t^{16}-5736 t^{12}+14849 t^{10}-470 t^{10}-1497 t^{8}\right.\right.$ \\
& $\left.\left.+196 t^{6}+95 t^{4}+2 t^{2}+1\right)\right) /\left((t-1)^{2}(t+1)^{2}\left(3 t^{2}+1\right)^{2}\left(8 t^{4}+t^{2}-1\right)^{2}\left(4 t^{2}-4 t^{4}+1\right)^{2}\right)$ \\
$140 a$ & $\left(-t^{2}\left(13440 t^{26}-503680 t^{24}+1587104 t^{22}-2412976 t^{20}+2149192 t^{18}-1169016 t^{16}+369280 t^{14}-47561 t^{12}-8778 t^{10}\right.\right.$ \\
& $\left.\left.+4313 t^{8}-492 t^{6}-39 t^{4}+14 t^{2}-1\right)\right) /\left((t-1)^{2}(t+1)^{2}\left(5 t^{2}-1\right)^{2}\left(8 t^{4}-5 t^{2}+1\right)^{2}\left(4 t^{6}+4 t^{4}-4 t^{2}+1\right)^{2}\right)$
\end{tabular}

\section{References}

1. D. Alexander, C. Cummins, J. McKay and C. Simons, 'Completely replicable functions', Groups, combinatorics \& geometry (Durham, 1990), London Mathematical Society Lecture Note Series 165 (Cambridge University Press, Cambridge, 1992) 87-98.

2. J. H. Conway, R. T. Curtis, S. P. Norton, R. A. Parker and R. A. Wilson, Atlas of finite groups. Maximal subgroups and ordinary characters for simple groups. With computational assistance from J. G. Thackray (Oxford University Press, Eynsham, 1985).

3. J. H. Conway and S. P. Norton, 'Monstrous moonshine', Bull. Lond. Math. Soc. 11 (1979) no. 3, 308-339.

4. R. Dedekind, 'Über Die Elliptischen Modul-Functionen', J. reine angew. Math. 3 (1877) no. 2, 265-292.

5. A. El Basraoui and A. Sebbar, 'Rational equivariant forms', Int. J. Number Theory 8 (2012) no. 4, 963-981.

6. D. Ford, Automorphic functions (Princeton University Press, 1951).

7. D. Ford, J. McKay and S. Norton, 'More on replicable functions', Comm. Algebra 22 (1994) no. 13, 5175-5193.

8. J. Harnard and J. McKay, 'Modular solutions to equations of generalized Halphen type', Proc. R. Soc. Lond. Ser. A Math. Phys. Eng. Sci. 456 (2000) no.1994, 261-294.

9. F. KLEIN, The icosahedron and the solution of equations of the fifth degree (Nauka, Moscow, 1989). Translated from the German by A. L. Gorodentsev and A. A. Kirillov. Translation edited and with a preface by A. N. Tyurin. With appendices by V. I. Arnold, J.-P. Serre and A. N. Tyurin.

10. J. MCKAY and A. Sebbar, 'Fuchsian groups, automorphic functions and Schwarzians', Math. Ann. 318 (2000) no. 2, 255-275.

11. V. Ovsienko and S. Tabachnikov, Projective differential geometry old and new. From the Schwarzian derivative to the cohomology of diffeomorphism groups, Cambridge Tracts in Mathematics 165 (Cambridge University Press, Cambridge, 2005).

12. H. Poincaré, 'Mémoire sur les fonctions fuchsiennes', Acta Math. 1 (1882) no. 1, 193-294 (French).

13. R. A. Rankin, Modular forms and functions (Cambridge University Press, 1977).

14. J.-P. Serre, A course in arithmetic, Graduate Texts in Mathematics 7 (Springer, New York-Heidelberg, 1973). Translated from the French.

\author{
Abdelkrim El Basraoui \\ Department of Mathematics and \\ Statistics \\ University of Ottawa \\ Ottawa \\ Ontario K1N6N5 \\ Canada \\ abdelkrim.elbasraoui@gmail.com
}

\author{
John McKay \\ Department of Mathematics and \\ Statistics \\ Concordia University \\ Montréal \\ Québec H3G 1M8 \\ Canada \\ mckay@encs.concordia.ca
}

